\title{
Solución numérica de las ecuaciones de Navier-Stokes por el método de descomposición de operadores
}

\author{
Tesis que presenta \\ Javier Salcedo Ruiz \\ Para obtener el grado de Maestro en Ciencias (Matemáticas) \\ Asesor \\ Dr. Francisco Javier Sánchez Bernabe
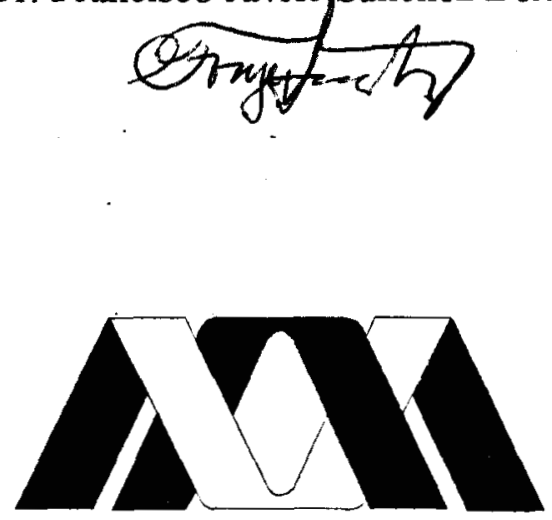 \\ Casa abierta al tiempo
}

Diciembre de 2000

UNIVERSIDAD AUTÓNOMA METROPOLITANA

UNIDAD IZTAPALAPA, División de Ciencias Básicas e Ingeniería. 


\section{ÍNDICE}

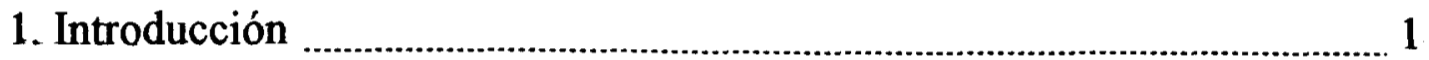

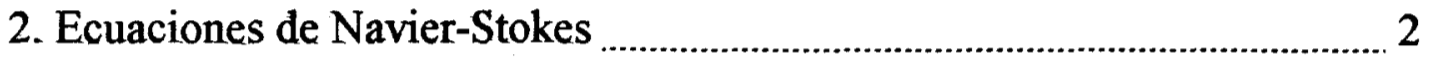

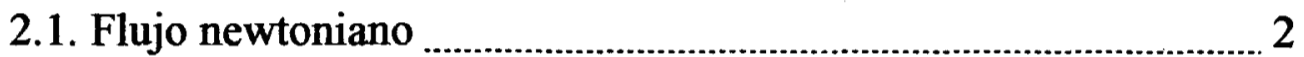

2.2. Obtención de las ecuaciones de Navier-Stokes ............................. 5

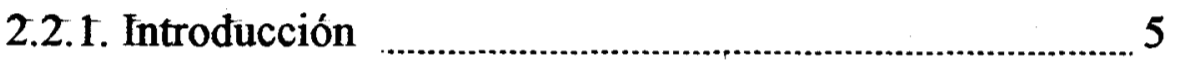

2.2.2. Condiciones de frontera tipo Dirichlet .............................. 9

2.2.3. Condiciones de frontera mixtas .......................................... 9

3. Problema variacional y método del elemento finito …….............................. 10

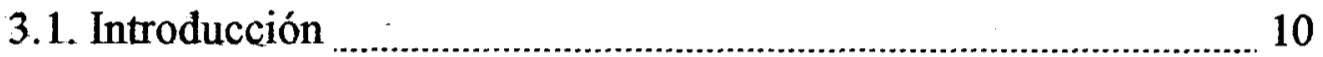

3.2. Formulación variacional de las ecuaciones de Navier-Stokes ...... 12

3.3. Espacio de discretización para el método del elemento finito ....... 15

4. Esquemas de descomposición de operadores ……....................................... 16

4.1. Esquema de Peaceman-Rachford ................................................... 16

4.2. Método teta

4.2.1. Caso de tres pasos .............................................................. 18

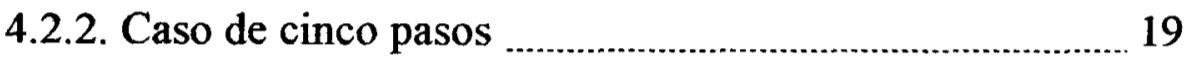

4.2.3. Aplicación a la simulación de un fluido newtoniano ...... 20 
4.3. Esquema de descomposición de operadores de primer orden ...... 22

4.3.1. Caso de descomposición en dos operadores ...................... 22

4.3.2. Caso de descomposición en tres operadores ................... 22

4.3.3. Determinación del orden del método ............................... 23

4.3.4. Aplicación a la simulación de un fluido newtoniano ...... 26

4.4. Método de Dyakonov .................................................................... 27

4.4.1. Caso de descomposición en dos operadores .................... 28

4.4.2. Caso de descomposición en tres operadores ..................... 29

4.4.3. Determinación del orden del método ……......................... 30

4.4.4. Aplicación a la simulación de un fluido newtoniano .......33

5. Solución del problema elíptico no lineal ...................................................... 34

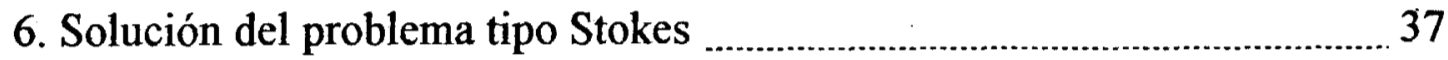

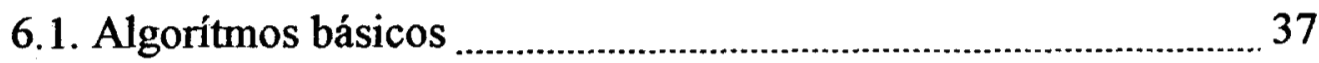

6.2. Esquema del elemento finito ……………………...................... 40

7. Resultados

7.1. Fluido en una cavidad con pared móvil .......................................... 43

7.2. Inyección doble en una cavidad cuadrada ....................................... 44

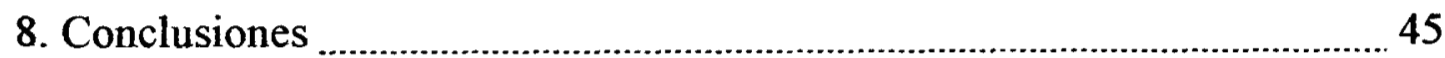

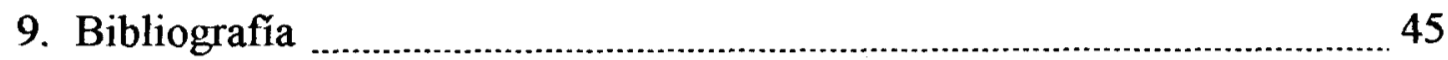




\section{SOLUCTÓN NUMÉRICA de las ECUACIONES de NAVIER STOKES por el MÉTODO de DESCOMPOSICIÓN de OPERADORES}

\section{Introducción}

Entre las áreas de la simulación numérica de fluidos, con mayor empuje, se encuentra el estudio de las ecuaciones de Navier-Stokes. La solución numérica de estas ecuaciones tiene una gran importancia en las aplicaciones industriales. Además, los métodos numéricos para resolverlas, con frecuencia, forman una parte básica de resolvedores para ecuaciones que modelan el flujo de fluidos más complejos o más generales como, por ejemplo, los fluidos nonewtonianos viscoplásticos de Bingham, los cuales tienen gran importancia en la industrià petrolèra, y los fluidos cuyo flujo esta modelado por la aproximación de Boussinesq.

El método de descomposición de operadores es una técnica muy poderosa en la resolución de ecuaciones diferenciales parciales dependientes del tiempo, especialmente de tipo nolineal o acopladas a ciertas restricciones, el cual permite resolver los problemas asociados con ellas, descomponiéndolos en varios problemas más simples. En la literatura se encuentra un buen número de este tipo de métodos aplicables a dos operadores. Sin embargo, el estudio de esquemas de descomposición de operadores aplicables a tres o más de ellos no es tan abundante. 
En este trabajo:

* Se estudia un método de descomposición de operadores, de primer orden, que permite descomponerlos en tres o más de ellos (el cual ya ha sido aplicado s la solución de las ecuaciones de Bingham), aplicándolo a la solución de las ecuaciones de Navier-Stokes.

* Se resuelve, con ese método el problema de la simulación del flujo de un fluido en una cavidad cuadrada cuya pared superior se desplaza a la derecha con velocidad unitaria.

* Con ese método de primer orden se resuelve además el problema de la inyección doble también en una cavidad cuadrada.

* Se resuelven los dos problemas antes referidos con el método teta.

* Se resuelven los subproblemas que resultan al aplicar estos métodos, utilizando un método de gradiente conjugado para los problemas de tipo Stokes, y un método de punto fijo para los problemas elípticos de tipo nolineal.

* Se estudia la eficiencia relativa del método de primer orden y del método teta, comparando la rapidez, con la que resuelven el problema de la cavidad cuadrada: También se determina que tan fina puede ser la malla espacial, y que tan grande se puede tomar la longitud del incremento del tiempo, de manera que se obtenga convergencia con esos métodos.

\section{Ecuaciones de Navier Stokes}

\subsection{Flujo newtoniano}

Dado el flujo de un fluido en una región $\Omega \subset R^{N}(N=2,3)$ conexa simple, abierta y con frontera $\Gamma$ de Lipschitz (suave por partes), con $\mathbf{x}=\left(x_{1}, x_{2}, x_{3}\right)$ se representará un punto cualquiera de $\Omega$ y con $\sigma=\left(\sigma_{i j}\right)$ se representará el tensor de esfuerzos cuyas componentes son 


$$
\boldsymbol{\sigma}=\left(\begin{array}{ccc}
\sigma_{11} & \tau_{12} & \tau_{13} \\
\tau_{12} & \sigma_{22} & \tau_{23} \\
\tau_{13} & \tau_{23} & \sigma_{33}
\end{array}\right)
$$

Los elementos de ese tensor que estan fuera de su diagonal principal (esfuerzos cortantes) estan asociados con las distorciones de los elementos (porciones) del fluido, y los elementos de la diagonal principal (esfuerzos normales) solamente cambian el volumen de los mismos sin alterar su forma y estan relacionados con la presión hidrostática $p$ del fluido. En realidad, puede demostrarse que la presión promedio en cada punto del fluido puede obtenerse mediante la siguiente expresión:

$$
\bar{p}=\frac{1}{3}\left(\sigma_{11}+\sigma_{22}+\sigma_{33}\right)
$$

Por lo anterior, se acostumbra descomponer el tensor de esfuersos en la siguiente forma:

$$
\boldsymbol{\sigma}=\left(\begin{array}{lll}
-p & 0 & 0 \\
0 & -p & 0 \\
0 & 0 & -p
\end{array}\right)+\left(\begin{array}{lll}
\sigma_{11}+p & \tau_{12} & \tau_{13} \\
\tau_{12} & \sigma_{22}+p & \tau_{23} \\
\tau_{13} & \tau_{23} & \sigma_{33}+p
\end{array}\right)
$$

o, usando una notación más compacta:

$$
\boldsymbol{\sigma}=-p \mathbf{I}+\boldsymbol{\sigma}^{\prime}
$$

en donde, $p=p(\mathbf{x}, t) \quad$ representa el campo escalar de presiones. $\mathrm{El}$ tensor $-p I$ determina los cambios de volumen de los elementos del fluido, mientras que el tensor $\boldsymbol{\sigma}^{\prime}$ determina los cambios de forma (distorsiones) de esos elementos.

Con $\mathbf{D}$ se representará el tensor de deformación cuyas componentes, en el caso $\Omega \subset R^{3}$, son: 


$$
\begin{aligned}
& \mathbf{D}=\frac{1}{2}\left(\nabla+\nabla^{T}\right) \mathbf{u}= \\
& =\left(\begin{array}{ccc}
\frac{\partial u_{1}}{\partial x_{1}} & \frac{1}{2}\left(\frac{\partial u_{1}}{\partial x_{2}}+\frac{\partial u_{2}}{\partial x_{1}}\right) & \frac{1}{2}\left(\frac{\partial u_{1}}{\partial x_{3}}+\frac{\partial u_{3}}{\partial x_{1}}\right) \\
\frac{1}{2}\left(\frac{\partial u_{2}}{\partial x_{1}}+\frac{\partial u_{1}}{\partial x_{2}}\right) & \frac{\partial u_{2}}{\partial x_{2}} & \frac{1}{2}\left(\frac{\partial u_{2}}{\partial x_{3}}+\frac{\partial u_{3}}{\partial x_{2}}\right) \\
\frac{1}{2}\left(\frac{\partial u_{3}}{\partial x_{1}}+\frac{\partial u_{1}}{\partial x_{3}}\right) & \frac{1}{2}\left(\frac{\partial u_{3}}{\partial x_{2}}+\frac{\partial u_{2}}{\partial x_{3}}\right) & \frac{\partial u_{3}}{\partial x_{3}}
\end{array}\right)
\end{aligned}
$$

en donde, en el caso en el que $\Omega \subset R^{3}, \quad \mathbf{u}=\mathbf{u}(\mathbf{x}, t)=\left\{u_{1}(\mathbf{x}, t), u_{2}(\mathbf{x}, t), u_{3}(\mathbf{x}, t)\right\}$ es el campo de velocidades del fluido. El tensor $D$ se puede escribir con ayuda del Jacobiano

$$
\nabla \mathbf{u}=\frac{\partial\left(u_{1}, u_{2}, u_{3}\right)}{\partial\left(x_{1}, x_{2}, x_{3}\right)}=\left(\begin{array}{ccc}
\frac{\partial u_{1}}{\partial x_{1}} & \frac{\partial u_{1}}{\partial x_{2}} & \frac{\partial u_{1}}{\partial x_{3}} \\
\frac{\partial u_{2}}{\partial x_{1}} & \frac{\partial u_{2}}{\partial x_{2}} & \frac{\partial u_{2}}{\partial x_{3}} \\
\frac{\partial u_{3}}{\partial x_{1}} & \frac{\partial u_{3}}{\partial x_{2}} & \frac{\partial u_{3}}{\partial x_{3}}
\end{array}\right)
$$

de la velocidad $\mathbf{u}, \mathbf{y}$ del tensor:

$$
\boldsymbol{\omega}=\left(\begin{array}{ccc}
0 & \frac{1}{2}\left(\frac{\partial u_{1}}{\partial x_{2}}-\frac{\partial u_{2}}{\partial x_{1}}\right) & -\frac{1}{2}\left(\frac{\partial u_{1}}{\partial x_{3}}-\frac{\partial u_{3}}{\partial x_{1}}\right) \\
-\frac{1}{2}\left(\frac{\partial u_{2}}{\partial x_{1}}-\frac{\partial u_{1}}{\partial x_{2}}\right) & 0 & \frac{1}{2}\left(\frac{\partial u_{2}}{\partial x_{3}}-\frac{\partial u_{3}}{\partial x_{2}}\right) \\
\frac{1}{2}\left(\frac{\partial u_{3}}{\partial x_{1}}-\frac{\partial u_{1}}{\partial x_{3}}\right) & -\frac{1}{2}\left(\frac{\partial u_{3}}{\partial x_{2}}-\frac{\partial u_{2}}{\partial x_{1}}\right) & 0
\end{array}\right)
$$


como sigue:

$$
\mathbf{D}=\nabla \mathbf{u}-\omega
$$

en donde, el tensor $\nabla \mathbf{u}$ incluye los cambios de volúmen mientras que $\omega$ incluye las distorsiones.

Se llama fluido newtoniano a cada fluido cuya ley constitutiva (fórmula que relaciona el tensor $\sigma^{\prime}$ con el tensor de deformaciones $\mathbf{D}$ ) tenga la siguiente forma:

$$
\sigma^{\prime}=2 \mu \mathrm{D}
$$

en donde $\mu$ representa la llamada viscocidad dinámica, misma que relaciona la velocidad relativa entre las capas del fluido con el esfuerzo cortante que aparece entre ellas.

Lo anterior implica que, en el caso de los fluidos newtonianos, el tensor de esfuerzos se puede escribir como sigue:

$$
\boldsymbol{\sigma}=-p \mathbf{I}+2 \mu \mathbf{D}=-p \mathbf{I}+\mu\left(\nabla+\nabla^{T}\right) \mathbf{u}
$$

Como se verá en el siguiente inciso, esta ecuación se requiere para obtener las ecuaciones que modelan los flujos de fluidos newtonianos.

\subsection{Obtención de las ecuaciones de Navier Stokes}

\subsubsection{Introducción}

Las ecuaciones de Navier Stokes ([8], pags. 141-162)

$$
\begin{gathered}
\frac{\partial \mathbf{u}}{\partial t}-\nu \Delta \mathbf{u}+(\mathbf{u} \cdot \nabla) \mathbf{u}+\nabla p=\mathbf{f}, \quad \text { en } Q=\Omega \times(0, T), \quad T>0, \quad(2.3)_{1} \\
\nabla \cdot \mathbf{u}=0 \quad \text { en } Q,
\end{gathered}
$$


modelan el flujo de los fluidos neutonianos, viscosos, incompresibles e isotérmicos. En el caso en el que $\Omega \subset R^{3}, \mathbf{f}=\mathbf{f}(\mathbf{x})=\left\{f_{1}(\mathbf{x}), f_{2}(\mathbf{x}), f_{3}(\mathbf{x})\right\}$ representa la llamada fuerza de volumen (fuerzas externas por unidad de masa) que actúa sobre el fluido y $(0, T)$ es el intervalo de tiempo durante el cual ocurre el flujo.

La ecuación (2.3) 2 se llama ecuación de continuidad y no es otra cosa que la forma diferencial de la ley de conservación de la masa aplicada al campo de velocidades de un fluido incompresible cualquiera.

La ecuación (2.3) 1 se obtiene a partir del principio de conservación del momento (segunda ley de Newton escrita en términos del momento lineal, según la cual, la suma de todas las fuerzas que actúan sobre una partícula es igual a la derivada temporal del momento lineal de la partícula); la cual, en el caso de de un sistema termodinámico cualquiera (porción de materia con fronteras bien definidas a través de las cuales no luye materia), limitado por la suferficie $\partial \Omega$ y con densidad $\rho$, toma la siguiente forma:

$$
\iiint_{\Omega} \rho \mathbf{f} d V+\iint_{\partial \boldsymbol{\Omega}} \boldsymbol{\sigma} \mathbf{n} d S=\frac{D}{D t} \iiint_{\Omega} \rho \mathbf{u} d V,
$$

en donde, la primera integral es igual a la fuerza total externa que actúa sobre el fluido, la segunda integral es igual a la fuerza total que actúa sobre la superficie del sistema (en ella, $\sigma$ representa el esfuerzo en la frontera del sistema y $\mathbf{n}$ representa la normal unitaria externa de la superficie $\partial \Omega$ ) y la tercera integral representa el momento lineal total del sistema. Aplicando el teorema de la divergencia a la integral superficial, se obtiene:

$$
\iint_{\partial \Omega} \boldsymbol{\sigma} \mathbf{n} d S=\iiint_{\Omega} \nabla \cdot \boldsymbol{\sigma} d V
$$

Lo cual, conjuntamente con la ecuación (2.4) implica lo siguiente:

$$
\iiint_{\Omega}\left[\frac{D(\rho \mathbf{u})}{D t}-\rho \mathbf{f}-\nabla \cdot \boldsymbol{\sigma}\right]=0
$$

Como la ecuación anterior se debe cumplir para todo sistema termodinámico, de ella se infiere lo siguiente (se supuso $\rho$ constante): 


$$
\rho \frac{D \mathbf{u}}{D t}-\rho \mathbf{f}-\nabla \cdot \boldsymbol{\sigma}=\mathbf{0}
$$

Tal ecuación es la llamada ecuación de Cauchy para fluidos incompresibles. Por otro lado, según la regla de la cadena, la aceleración a del fluido (derivada respecto al tiempo de la velocidad $\left.\mathbf{u}=\mathbf{u}(\mathbf{x}, t)=\left\{u_{1}(\mathbf{x}, t), u_{2}(\mathbf{x}, t), u_{3}(\mathbf{x}, t)\right\}\right)$ se obtiene como sigue:

$$
\begin{gathered}
\mathbf{a}=\frac{D}{D t} \mathbf{u}\left(x_{1}, x_{2}, x_{3}, t\right)=\frac{\partial \mathbf{u}}{\partial t}+\frac{\partial \mathbf{u}}{\partial x_{1}} \frac{d x_{1}}{d t}+\frac{\partial \mathbf{u}}{\partial x_{2}} \frac{d x_{2}}{d t}+\frac{\partial \mathbf{u}}{\partial x_{3}} \frac{d x_{3}}{d t}= \\
=\frac{\partial \mathbf{u}}{\partial t}+u_{1} \frac{\partial \mathbf{u}}{\partial x_{1}}+u_{2} \frac{\partial \mathbf{u}}{\partial x_{2}}+u_{3} \frac{\partial \mathbf{u}}{\partial x_{3}}= \\
=\frac{\partial \mathbf{u}}{\partial t}+\mathbf{u} \cdot\left(\frac{\partial\left(u_{1}, u_{2}, u_{3}\right)}{\partial\left(x_{1}, x_{2}, x_{3}\right)}\right)=(\mathbf{u} \cdot \nabla) \mathbf{u}+\frac{\partial \mathbf{u}}{\partial t}
\end{gathered}
$$

Si se sustituye lo anterior en la ecuación (2.7) y se toma en cuenta (2.2), se obtiene:

$$
\begin{gathered}
\rho \frac{D \mathbf{u}}{D t}=\rho\left[(\mathbf{u} \cdot \nabla) \mathbf{u}+\frac{\partial \mathbf{u}}{\partial t}\right] \\
=\rho \mathbf{f}+\nabla \cdot \boldsymbol{\sigma}=\rho \mathbf{f}+\nabla \cdot\left(-p \mathbf{I}+\mu\left(\nabla \mathbf{u}+\nabla^{T} \mathbf{u}\right)\right)= \\
=\rho \mathbf{f}-\nabla p+\mu \nabla \cdot \nabla \mathbf{u}+\nabla \cdot(\nabla \mathbf{u})^{T}= \\
=\rho \mathbf{f}-\nabla p+\mu \Delta \mathbf{u}
\end{gathered}
$$

Para obtener lo anterior se tomó en cuenta lo siguiente: 


$$
\begin{gathered}
\nabla \cdot(p \mathbf{I})=\nabla \cdot\left(\begin{array}{lll}
p & 0 & 0 \\
0 & p & 0 \\
0 & 0 & p
\end{array}\right)=\left(\begin{array}{ccc}
\frac{\partial p}{\partial x_{1}} & 0 & 0 \\
0 & \frac{\partial p}{\partial x_{2}} & 0 \\
0 & 0 & \frac{\partial p}{\partial x_{3}}
\end{array}\right)=\nabla p \\
\nabla \cdot(\nabla \mathbf{u})=\nabla \cdot\left(\begin{array}{ccc}
\frac{\partial u_{1}}{\partial x_{1}} & \frac{\partial u_{1}}{\partial x_{2}} & \frac{\partial u_{1}}{\partial x_{3}} \\
\frac{\partial u_{2}}{\partial x_{1}} & \frac{\partial u_{2}}{\partial x_{2}} & \frac{\partial u_{2}}{\partial x_{3}} \\
\frac{\partial u_{3}}{\partial x_{1}} & \frac{\partial u_{3}}{\partial x_{2}} & \frac{\partial u_{3}}{\partial x_{3}}
\end{array}\right)=\left(\begin{array}{c}
\nabla \cdot\left(\nabla u_{1}\right) \\
\nabla \cdot\left(\nabla u_{2}\right) \\
\nabla \cdot\left(\nabla u_{3}\right)
\end{array}\right)=\left(\begin{array}{c}
\Delta u_{1} \\
\Delta u_{2} \\
\Delta u_{3}
\end{array}\right)=\Delta \mathbf{u} \\
\left(\begin{array}{lll}
\frac{\partial u_{1}}{\partial x_{1}} & \frac{\partial u_{2}}{\partial x_{1}} & \frac{\partial u_{3}}{\partial x_{1}} \\
\frac{\partial u_{1}}{\partial x_{2}} & \frac{\partial u_{2}}{\partial x_{2}} & \frac{\partial u_{3}}{\partial x_{2}} \\
\frac{\partial u_{1}}{\partial x_{3}} & \frac{\partial u_{2}}{\partial x_{3}} & \frac{\partial u_{3}}{\partial x_{3}}
\end{array}\right)=\left(\begin{array}{l}
\frac{\partial(\nabla \cdot \mathbf{u})}{\partial x_{1}} \\
\frac{\partial(\nabla \cdot \mathbf{u})}{\partial x_{2}} \\
\frac{\partial(\nabla \cdot \mathbf{u})}{\partial x_{3}}
\end{array}\right)=\left(\begin{array}{l}
0 \\
0 \\
0 \\
0
\end{array}\right)
\end{gathered}
$$

La última igualdad es consecuencia de $(2.3)_{2}$. De (2.9), después de reordenar términos y dividir entre $\rho$, se obtiene:

$$
\frac{\partial \mathbf{u}}{\partial t}-\frac{\mu}{\rho} \Delta \mathbf{u}+(\mathbf{u} \cdot \nabla) \mathbf{u}+\frac{1}{\rho} \nabla p=\mathbf{f}
$$

Si se considera que $\frac{\mu}{\rho}=\nu$ es la llamada viscocidad cinemática y se sustiyuye $p$ por $\frac{p}{\rho}$, se obtiene finalmente $(2.3)_{1}$. 
Introduciendo como unidades para medir distancia y velocidad, una distancia $L$ y una velocidad $V$, características del problema de que se trate (lo cual determina como unidad para medir tiempo $T=L / V$ ), y haciendo los cambios de variables $\mathbf{u}^{\prime}=\mathbf{u} / V, \mathbf{x}^{\prime}=\mathbf{x} / L$ y $t^{\prime}=t / T$, se obtienen las ecuaciones de Navier-Stokes en forma adimensional (independiente de las unidades de medición), si en ellas se sustituye $\nu$ con el llamado número de Reynolds:

$$
R e=\frac{L U}{\nu}
$$

La modelación del flujo de un fluido determinado requiere, además de las ecuaciones (2.3), del establecimiento de condiciones iniciales

$$
\mathbf{u}(\mathbf{x}, 0)=\mathbf{u}_{\mathbf{0}}(\mathbf{x}), \quad \text { para todo } \quad \mathbf{x} \in \boldsymbol{\Omega}, \quad \text { con } \nabla \cdot \mathbf{u}_{\mathbf{0}}=0
$$

y de condiciones de frontera convenientes. Los siguientes tipos de condiciones de frontera son de especial interés:

\subsubsection{Condiciones de frontera tipo Dirichlet}

$$
\mathbf{u}=\mathbf{g}(\mathbf{x}, t) \quad \text { para } \mathbf{x} \in \Gamma \quad \mathbf{y} \quad t>0
$$

\subsubsection{Condiciones de frontera mixtas}

$$
\begin{gathered}
\mathbf{u}=\mathbf{g}_{\mathbf{0}}(\mathbf{x}, t), \quad \text { para } \mathbf{x} \in \Gamma_{0} \quad \mathbf{y} \quad t>0 \\
\nu \frac{d \mathbf{u}}{d \mathbf{n}}-\mathbf{n} p=\mathbf{g}_{1} \quad \text { para } \mathbf{x} \in \Gamma_{1} \quad \mathbf{y} \quad t>0
\end{gathered}
$$


en donde $\Gamma_{0}$ y $\Gamma_{1}$ representan dos subconjuntos disjuntos de $\Gamma$ tales que $\Gamma_{0} \cup \Gamma_{1}=\Gamma, \quad \mathbf{n}$ representa la normal unitaria exterior de $\Gamma$ y la derivada en la dirección de $\mathbf{n}$ está dada por

$$
\frac{d \mathbf{u}}{d \mathbf{n}}=\left(\nabla u_{1} \cdot \mathbf{n}, \nabla u_{2} \cdot \mathbf{n}, \nabla u_{3} \cdot \mathbf{n}\right)
$$

En el caso en el que ni entra ni sale fluido a través de $\Gamma$, son suficientes las condiciones de frontera tipo Dirichlet. En toda pared impermeable $\mathbf{u} \cdot \mathbf{n}=\mathbf{0}$. Si la pared está en reposo, por exigencias de la teoría de la capa límite de fluidos viscosos (condición de no deslizamiento de fluidos viscosos), $\mathbf{u} \cdot \mathbf{t}=\mathbf{0}$ ( $t$ representa el vector unitario de la tangente de la frontera). Por otro lado, si la frontera es impermeable y se encuentra en reposo $u=0, y$ si la pared es impermeable y está en movimiento $\mathbf{u}=\mathbf{g}_{0}$.

\section{Problema variacional y método del elemento finito}

\subsection{Introducción}

Se llama problema variacional, a cada problema que consista en encontrar la función $u_{0}$ en la cual un funcional determinado $F: V \rightarrow R, F=F(u)$ (en donde $V$ es algún espacio de funciones), adquiere características determinadas (como tener su valor mínimo, por ejemplo); o la función $u_{0}$ que hace que un funcional determinado $H: V \times V \rightarrow R, H=H(u, v)$, sea igual a otro funcional $G: V \rightarrow R, G=G(v)$, para cada $v \in V$. Muchos problemas de ecuaciones diferenciales se pueden replantear, en forma equivalente, como problemas variacionales. La reformulación de los problemas de ecuaciones diferenciales como problemas equivalentes de la segunda forma antes mencionada, se llama formulación débil de los mismos, cuando tales problemas no admiten ser planteados también en la primera forma. 
El espacio $V$ de las formulaciones variacionales y débiles es, en general, de dimensión infinita (las funciones de $V$ no se pueden definir mediante una cantidad finita de variables), en cuyo caso, tales problemas no siempre se pueden resolver en forma exacta. Se llama método del elemento finito a un método que permite buscar soluciones aproximadas y discretas, de las formulaciones variacionales y débiles, de problemas de ecuaciones diferenciales, dentro de espacios $V_{h}$ de dimensión finita (un espacio de polinomios).

Por ejemplo, se puede demostrar que el problema que consiste en encontrar una función $u \in V$ que tenga segunda derivada continua y satisfaga el siguiente problema de frontera:

$$
\begin{aligned}
u^{\prime \prime}(x) & =f(x), \quad 0<x<1 \\
u(0) & =u(1)=0,
\end{aligned}
$$

es equivalente al problema de minimización que consiste en encontrar $u \in V$ tal que, para el funcional

$$
F: V \rightarrow R, \quad F(v)=\frac{1}{2}\left(v^{\prime}, v^{\prime}\right)-(f(v)-v), \quad(v, u)=\int_{0}^{1} v(x) u(x) d x
$$

se cumpla que

$$
F(u) \leq F(v) \quad \text { para todo } v \in V
$$

y es equivalente también al problema variacional que consiste en encontrar $u \in V \quad$ tal que

$$
\left(u^{\prime}, v^{\prime}\right)=(f, v) \quad \text { para todo } v \in V
$$

Figure 1: 
Para encontrar una solución aproximada y discreta de esos problemas (es decir para encontrar los valores aproximados de $u$ en un conjunto discreto de $M+1$ puntos del intervalo $[0,1])$, por el método del elemento finito ([4]), usando funciones de interpolación lineales por trozos (en el caso general se utilizan polinomios), se procede como sigue: mediante $M+2$ puntos cualesquiera $x_{0}, x_{1}, \ldots x_{M}, x_{M \mid 1}$, se divide ese intervalo en $M+$ 1 subintervalos $\quad I_{j}:=\left(x_{j-1}, x_{j}\right) \quad$ de medidas $h_{j}:=x_{j}-$ $x_{j-1}, \quad j=1,2, \ldots, M+1, \quad$ respectivamente; luego se define el espacio de funciones

$V_{h}:=\left\{\begin{array}{c}v: v \text { es lineal sobre cada subintervalo } I_{j}, \text { es continua sobre }[0,1] \\ \text { y satisface } \quad v(0)=v(1)=0\end{array}\right\}$

en donde $\left(h:=\max _{1 \leq j \leq M+1}\left\{h_{j}\right\}\right)$, y se resuelve el problema variacional aproximado discreto (método de Ritz) que consiste en encontrar $u_{h} \in V_{h}$ tal que

$$
F\left(u_{h}\right) \leq F(v) \quad \text { para todo } v \in V_{h}
$$

o se resuelve el problema variacional aproximado discreto (método de Garlekin) que consiste en encontrar $u_{h} \in V_{h}$ tal que

$$
\left(u_{h}^{\prime}, v^{\prime}\right)=(f, v) \quad \text { para toda } v \in V_{h} .
$$

Como el conjunto de funciones

$$
\varphi_{j} \in V_{h}, \quad j=1, \ldots, M,
$$

tales qué

$$
\varphi_{j}\left(x_{i}\right)=\left\{\begin{array}{lll}
1 & \text { si } & i=j \\
0 & \text { si } & i \neq j
\end{array}\right\}
$$

constituye una base del espacio vectorial $V_{h}$, cualquiera de los dos problemas aproximados antes referidos se reduce al problema de resolver un sistema de ecuaciones lineales. Los problemas de ecuaciones diferenciales se pueden resolver numéricamente utilizando métodos de diferencias finitas ([7]). Aquí se resolvieron mediante el método del elemento finito porque, aunque este método es más difícil de aplicar, en general, es más poderoso. 


\subsection{Formulación variacional de las ecuaciones de Navier- Stokes}

Definiendo los siguientes espacios de tipo Sobolev, para el caso en el que $\Omega \subset R^{2}$ :

$$
\begin{gathered}
\mathbf{V}_{\mathbf{0}}=\left\{\mathbf{u} \mid \mathbf{u} \in H^{1}(\Omega)^{2}, \mathbf{u}=\mathbf{0} \text { sobre } \Gamma_{0}\right\}=H_{0}^{1}(\Omega)^{2} \\
\mathbf{V}_{g}=\left\{\mathbf{u} \mid \mathbf{u} \in H^{1}(\Omega)^{2}, \mathbf{u}=\mathbf{g}_{0} \text { sobre } \Gamma_{0}\right\}
\end{gathered}
$$

en donde

$$
\begin{gathered}
H^{1}(\Omega)=\left\{u: \Omega \rightarrow R \mid u, \quad \frac{\partial u}{\partial x_{i}} \in L^{2}(\Omega), \quad i=1,2\right\}, \\
H_{0}^{1}(\Omega)=\left\{u \in H^{1}(\Omega) \mid u=0 \text { sobre } \Gamma\right\}
\end{gathered}
$$

Si $g_{0}$ es suficientemente suave, entonces $\mathrm{V}_{g}$ no es vacío $\mathrm{y}$, mediante el uso de la notación

$$
d x=d x_{1} d x_{2},
$$

se puede probar que: para toda funcion $\mathbf{u} \in H^{1}(\Omega)^{2}=H^{1}(\Omega) \times H^{1}(\Omega)$, toda función $\mathbf{v} \in \mathrm{V}_{\mathbf{0}} \mathrm{y}$ para toda $p \in L^{2}(\Omega)$, se cumplen las llamadas fórmulas (identidades) de Green:

$$
\begin{aligned}
& \int_{\boldsymbol{\Omega}} \Delta \mathbf{u} \cdot \mathbf{v} d x=\int_{\Gamma_{1}} \frac{\partial \mathbf{u}}{\partial n} \cdot \mathbf{v} d \Gamma-\int_{\boldsymbol{\Omega}} \nabla \mathbf{u} \cdot \nabla \mathbf{v} d x \\
& \int_{\mathbf{\Omega}} \nabla p \cdot \mathbf{v} d x=\int_{\Gamma_{1}} p \mathbf{n} \cdot \mathbf{v} d \Gamma-\int_{\Omega} p \nabla \cdot \mathbf{v} d x
\end{aligned}
$$

en donde:

$$
\nabla \cdot \mathbf{u}=\frac{\partial u_{1}}{\partial x_{1}}+\frac{\partial u_{2}}{\partial x_{2}}
$$




$$
\nabla \mathbf{u}=\left(\begin{array}{ll}
\frac{\partial u_{1}}{\partial x_{1}} & \frac{\partial u_{1}}{\partial x_{2}} \\
\frac{\partial u_{2}}{\partial x_{1}} & \frac{\partial u_{2}}{\partial x_{2}}
\end{array}\right) \quad \text { y } \quad \nabla \mathbf{u} \cdot \nabla \mathbf{v}=\nabla u_{1} \cdot \nabla v_{1}+\nabla u_{2} \cdot \nabla v_{2}
$$

Observese que la región de integración de una de las integrales es solamente una parte $\Gamma_{1}$ de $\Gamma$, porque $v$ pertenece a $V_{0}$.

Para obtener una formulación variacional de las ecuaciones (2.3) con las condiciones de frontera (2.12), se multiplica (2.3), por una función de prueba cualquiera $v \in V_{0}$ y se integra sobre $\Omega$, para obtener ([2], pags. 416-431):

$$
\int_{\Omega} \frac{\partial \mathbf{u}}{\partial t} \cdot \mathbf{v} d x-\nu \int_{\Omega} \Delta \mathbf{u} \cdot \mathbf{v} d x+\int_{\Omega}(\mathbf{u} \cdot \nabla) \mathbf{u} \cdot \mathbf{v} d \Gamma-\int_{\Omega} \nabla p \cdot \mathbf{v} d x=\int_{\Omega} \mathbf{f} \cdot \mathbf{v} d x
$$

Usando (3.6) y (3.7) :

$$
\begin{aligned}
\int_{\Omega} \frac{\partial \mathbf{u}}{\partial t} \cdot \mathbf{v} d x & +\nu \int_{\Omega} \nabla \mathbf{u} \cdot \nabla \mathbf{v} d x-\nu \int_{\Gamma_{1}} \frac{\partial \mathbf{u}}{\partial n} \cdot \mathbf{v} d \Gamma+\int_{\Omega}(\mathbf{u} \cdot \nabla) \mathbf{u} \cdot \mathbf{v} d \Gamma+ \\
& +\int_{\Gamma_{1}} p \mathbf{n} \cdot \mathbf{v} d \Gamma-\int_{\Omega} p \nabla \cdot \mathbf{v} d x=\int_{\Omega} \mathbf{f} \cdot \mathbf{v} d x
\end{aligned}
$$

Así que, multiplicando (2.3) ${ }_{2}$ por $q \in L^{2}(\Omega)$ e integrando, y tomando en cuenta las condiciones de frontera (2.12), se infiere que la velocidad $\mathbf{u}$ y la presión $p$ que satisfacen las ecuaciones (2.3) con las condiciones de frontera (2.12), satisfacen también la siguiente formulación débil de ese problema:

$$
\begin{gathered}
\text { encontrar } \quad\{\mathbf{u}(t), p(t)\} \in \mathbf{V}_{g} \times L^{2}(\Omega), \quad \text { tal que } \\
\forall \mathbf{v} \in \mathbf{V}_{\mathbf{0}} \quad \text { y } \quad \forall q \in H^{1}(\Omega), \quad \text { se cumpla: }
\end{gathered}
$$




$$
\begin{gathered}
\int_{\Omega} \frac{\partial \mathbf{u}}{\partial t} \cdot \mathbf{v} d x+\nu \int_{\Omega} \nabla \mathbf{u} \cdot \nabla \mathbf{v} d x+\int_{\mathbf{\Omega}}(\mathbf{u} \cdot \nabla) \mathbf{u} \cdot \mathbf{v} d \Gamma-\int_{\mathbf{\Omega}} p \nabla \cdot \mathbf{v} d x= \\
=\int_{\Omega} \mathbf{f} \cdot \mathbf{v} d x+\int_{\Gamma_{1}} \mathbf{g}_{1} \cdot \mathbf{v} d \Gamma \\
\int_{\Omega} q \nabla \cdot \mathbf{u} d x=0
\end{gathered}
$$

La formulación débil anterior vale para todo $t>0$. Más adelante se mostrará como discretizar la derivada temporal de u en la ecuación (3.10)

\subsection{Espacio de discretización para el método del ele- mento finito}

Dada una región cualquiera $\Omega$ de $R^{2}$ limitada por una línea poligonal y dada una triangulación cualquiera $\mathcal{T}_{h}$ de esa región para elemento finito (conjunto de triángulos $K_{1}, K_{2}, \ldots, K_{n}$, que sin traslaparse y sin que el vértice de alguno de ellos descanse en el lado de otro, cumplen $\Omega=K_{1} \cup K_{2} \cup \ldots \cup K_{n}$; para ello, si es necesario, $\Gamma$ se aproxima mediante una poligonal. $h$ representa la longitud máxima de los triángulos de esa triangulación), si con $\mathcal{P}_{k}$ se representa el espacio de todos los polinomios de grado menor o igual que $k$; entonces se definen los siguientes conjuntos:

$$
\begin{gathered}
H_{h}^{1}=\left\{\psi_{h} \in C^{0}(\bar{\Omega})\left|\psi_{h}\right|_{T} \in \mathcal{P}_{1}, \forall T \in \mathcal{T}_{h}\right\} \\
\mathbf{V}_{h}=\left\{\mathbf{v}_{h}\left|\mathbf{v}_{h} \in C^{0}(\bar{\Omega}) \times C^{0}(\bar{\Omega}), \overline{\mathbf{v}}_{h}\right|_{T} \in \mathcal{P}_{1} \times \mathcal{P}_{1} \forall T \in \overline{\mathcal{T}_{h}}\right\} \\
\mathbf{V}_{g h}=\left\{\mathbf{v}_{h} \in \mathbf{V}_{h} \mid \mathbf{v}_{h}=\mathbf{g}_{h} \text { sobre } \Gamma_{\mathbf{0}}\right\} \\
\mathbf{V}_{0 h}=\left\{\mathbf{v}_{h} \in \mathbf{V}_{h} \mid \mathbf{v}_{h}=0 \text { sobre } \Gamma_{0}\right\}
\end{gathered}
$$

en dode, $\overline{\mathcal{T}_{h}}$ es la triangulación de $\Omega$ obtenida a partir de $\mathcal{T}_{h}$ al unir con rectas los puntos medios de los lados de cada $T \in \mathcal{T}_{h}$. Los espacios $\mathbf{V}_{g h}, \mathbf{V}_{\mathbf{0} h}$ y $H_{h}^{1}$, aproximan los espacios $\mathbf{V}_{g}, \mathbf{V}_{\mathbf{0}}$ y $H^{1}$, respectivamente, al aplicar el método del elemento finito (véanse (3.1), (3.2) y (3.3)). 


\section{Esquemas de descomposición de operadores para discretización del tiempo}

El método de descomposición de operadores es una técnica muy poderosa en la resolución de ecuaciones diferenciales parciales dependientes del tiempo, especialmente de tipo nolineal o acopladas a ciertas restricciones, el cual permite resover los problemas asociados con ellas, descomponiéndolos en varios problemas más simples. En seguida se describen algunos de los esquemas de descomposición de operadores, más importantes ([6], pags. 113-126).

\subsection{Esquema de Peaceman-Rachford}

Dado el siguiente problema de valor inicial con condiciones de frontera tipo Dicichlet ([3], pags. 222-226):

$$
\begin{gathered}
\mathbf{u}_{t}+A(\mathbf{u})=\mathbf{f} \quad \text { en } \quad \Omega \times(0, T), \quad T>0, \\
\mathbf{u}(x, 0)=\mathbf{u}_{0} \\
\mathbf{u}=\mathbf{g} \quad \text { sobre } \Gamma,
\end{gathered}
$$

en dondè $\Omega \subset R^{2}$, A es un operador de un espacio de Hilbert $H$ en él mismo, formado solamente por operadores de derivación espacial, $\mathbf{u}_{0} \in H$ representa al valor inicial de $\mathbf{u}$ y $\mathbf{f}$ representa el término fuente; si $\mathrm{A}$ se puede descomponer en la suma de dos operadores $A_{1}$ y $A_{2}$, tales que

$$
A=A_{1}+A_{2}
$$

entonces el método de Peaceman Rachford proporciona un esquema de discretización en el tiempo, de dos pasos, definido de la manera siguiente: 
Cada intervalo de tiempo $[n \Delta t,(n+1) \Delta t]$ se divide en dos subintervalos de igual longitud $\Delta t / 2$. Luego, dado $\mathbf{u}^{0}=\mathbf{u}_{0}$, para cada $n \geq 0$, con $\mathbf{u}^{n}$ conocido, calcular sucesivamente $\mathbf{u}^{n+\frac{1}{2}}$ y $\mathbf{u}^{n+1}$, en donde $\mathbf{u}^{k}=\mathbf{u}(x, k \Delta t)$, mediante:

$$
\begin{gathered}
\frac{\mathbf{u}^{n+\frac{1}{2}}-\mathbf{u}^{n}}{\Delta t / 2}+A_{1}\left(\mathbf{u}^{n+\frac{1}{2}}\right)+A_{2}\left(\mathbf{u}^{n}\right)=\mathbf{f}^{n+\frac{1}{2}}, \\
\mathbf{u}^{n+\frac{1}{2}}=\mathbf{g}^{n+\frac{1}{2}} \quad \text { sobre } \Gamma \\
\mathbf{y} \\
\frac{\mathbf{u}^{n+1}-\mathbf{u}^{n+\frac{1}{2}}}{\Delta t / 2}+A_{1}\left(\mathbf{u}^{n+\frac{1}{2}}\right)+A_{2}\left(\mathbf{u}^{n+1}\right)=\mathbf{f}^{n+1}, \\
\mathbf{u}^{n+1}=\mathbf{g}^{n+1} \quad \text { sobre } \Gamma,
\end{gathered}
$$

en donde $\Delta t$ (positivo) representa el paso de discretización del tiempo, $\mathbf{u}^{n+q}$ representa una aproximación de $\mathbf{u}[(n+q) \Delta t]$ y $\mathbf{f}^{n+q}=\mathbf{f}[(n+q) \Delta t]$. Como puede verse, una vez que se divide el operador $A$ y que se divide el intervalo de tiempo. $[n \Delta t,(n+1) \Delta t]$ en dos subintervalos de igual longitud $\Delta t / 2$, en el primer subintervalo la aplicación de $A_{1}$ se hace implicitamente y la de $A_{2}$ explícitamente $\mathrm{y}$, luego, en el segundo subintervalo se invierte esa forma de aplicación de $A_{1} \mathrm{y}$ de $A_{2}$.

Este esquema es de dos pasos, no permite descomponer el operador $A$ en más de dos operadores $\mathrm{y}$, aunque es de segundo orden $\mathrm{e}$ incondicionablemente estable, no siempre permite obtener las soluciones estacionarias.

\subsection{Método teta}

Este método fue ideado para evitar la debilidad del método de PeacemanRachford para obtener soluciones estacionarias. A diferencia del esquema de Peaceman-Rachford, el cual es de dos pasos, el método teta puede ser de tres o de cinco pasos ([3], 228-232). 


\subsubsection{Caso de tres pasos}

En este caso, el método teta requiere de tres constantes cuyos valores

$$
\Theta=\frac{2-\sqrt{2}}{2}, \quad \alpha=2-\sqrt{2} \quad \text { y } \quad \beta=\sqrt{2}-1
$$

se determinan de tal manera que se consiga, entre otras cosas, que el método sea de segundo orden de precisión; y, para resolver el problema de valor inicial (4.1), el operador $A$ se descompone en la suma de dos operadores $A_{1}$ y $A_{2}$, en la siguiente forma:

$$
A=\alpha A_{1}+\beta A_{2},
$$

después, cada intervalo de tiempo $[n \Delta t,(n+1) \Delta t]$ se divide en tres subintervalos, dos de igual longitud $\Theta \Delta t$ (el primero y el último) y otro de longitud $(1-2 \theta)$. Luego, dado $\mathbf{u}^{0}=\mathbf{u}_{0}$, para cada $n \geq 0$, con $\mathbf{u}^{n}$ conocido, calcular sucesivamente $\mathbf{u}^{n+\Theta}, \mathbf{u}^{n+1-\Theta}$ y $\mathbf{u}^{n+1}$ mediante:

$$
\begin{gathered}
\frac{\mathbf{u}^{n+\Theta}-\mathbf{u}^{n}}{\Theta \Delta t}+A_{1}\left(\mathbf{u}^{n+\Theta}\right)+A_{2}\left(\mathbf{u}^{n}\right)=\mathbf{f}^{n+\Theta}, \\
\mathbf{u}^{n+\Theta}=\mathbf{g}^{n+\Theta} \quad \text { sobre } \Gamma, \\
\frac{\mathbf{u}^{n+1-\Theta}-\mathbf{u}^{n+\Theta}}{(1-2 \Theta) \Delta t}+A_{2}\left(\mathbf{u}^{n+1-\Theta}\right)+A_{1}\left(\mathbf{u}^{n+\Theta}\right)=\mathbf{f}^{n+1-\Theta}, \\
\mathbf{u}^{n+1-\Theta}=\mathbf{g}^{n+1-\Theta} \quad \text { sobre } \Gamma, \\
\frac{\mathbf{u}^{n+1}-\mathbf{u}^{n+1-\Theta}}{\Theta \Delta t}+A_{1}\left(\mathbf{u}^{n+1}\right)+A_{2}\left(\mathbf{u}^{n+1-\Theta}\right)=\mathbf{f}^{n+1}, \\
\mathbf{u}^{n+1}=\mathbf{g}^{n+1} \quad \text { sobre } \Gamma,
\end{gathered}
$$

en donde $\Delta t$ (positivo) representa el paso de discretización del tiempo, $\mathbf{u}^{n+q}$ representa una aproximación de $\mathbf{u}[(n+q) \Delta t] \quad$ y $\mathbf{f}^{n+q}=\mathbf{f}[(n+q) \Delta t]$. Este esquema es de tres pasos y no permite descomponer el operador $A$ en más de dos operadores, es de segundo orden en el tiempo e incondicionablemente estable y permite capturar las soluciones estacionarias. 


\subsubsection{Caso de cinco pasos}

En este caso:

$$
\Theta=1-\frac{\sqrt{6}}{3}, \quad \Theta^{\prime}=\frac{1-3 \Theta}{2}, \quad \alpha=\frac{3 \Theta-1}{\Theta-1} \quad \text { y } \quad \beta=1-\alpha
$$

cada intervalo de tiempo $[n \Delta t,(n+1) \Delta t]$ se divide en cinco subintervalos de longitudes: $\Theta \Delta t, \Theta^{\prime} \Delta t, \Theta \Delta t, \Theta^{\prime} \Delta t, \Theta \Delta t$; luego, dado $\mathbf{u}^{\mathbf{0}}=\mathbf{u}_{\mathbf{0}}$, para cada $n \geq 0$, con $\mathbf{u}^{n}$ conocido, calcular sucesivamente $\mathbf{u}^{n+\Theta}, \mathbf{u}^{n+\theta+\theta^{\prime}}$, $\mathbf{u}^{n+2 \theta+\Theta^{\prime}}, \mathbf{u}^{n+1-\theta}$ y $\mathbf{u}^{n+1}$ mediante:

$$
\begin{aligned}
& \frac{\mathbf{u}^{n+\Theta}-\mathbf{u}^{n}}{\Theta \Delta t}+A_{1}\left(\mathbf{u}^{n+\Theta}\right)+A_{2}\left(\mathbf{u}^{n}\right)=\mathbf{f}^{n+\Theta}, \\
& \mathbf{u}^{n+\theta}=\mathbf{g}^{n+\Theta} \quad \text { sobre } \Gamma \\
& \frac{\mathbf{u}^{n+\Theta+\boldsymbol{\Theta}^{\prime}}-\mathbf{u}^{n+\Theta}}{\Theta^{\prime} \Delta t}+A_{2}\left(\mathbf{u}^{n+\boldsymbol{\theta}+\boldsymbol{\Theta}^{\prime}}\right)+A_{1}\left(\mathbf{u}^{n+\Theta}\right)=\mathbf{f}^{n+\boldsymbol{\theta}+\boldsymbol{\Theta}^{\prime}}, \\
& \mathbf{u}^{n+\Theta+\Theta^{\prime}}=\mathbf{g}^{n+\theta+\Theta^{\prime}} \quad \text { sobre } \Gamma, \\
& \frac{\mathbf{u}^{n+2 \Theta+\Theta^{\prime}}-\mathbf{u}^{n+\Theta+\Theta^{\prime}}}{\Theta \Delta t}+A_{1}\left(\mathbf{u}^{n+2 \Theta+\Theta^{\prime}}\right)+A_{2}\left(\mathbf{u}^{n+\Theta+\Theta^{\prime}}\right)=\mathbf{f}^{n+2 \Theta+\Theta^{\prime}} \\
& \mathbf{u}^{n+2 \theta+\boldsymbol{\theta}^{\prime}}=\mathbf{g}^{n+2 \theta+\boldsymbol{\Theta}^{\prime}} \quad \text { sobre } \Gamma, \\
& \frac{\mathbf{u}^{n+1-\Theta}-\mathbf{u}^{n+2 \Theta+\Theta^{\prime}}}{\Theta^{\prime} \Delta t}+A_{2}\left(\mathbf{u}^{n+1-\Theta}\right)+A_{1}\left(\mathbf{u}^{n+2 \Theta+\Theta^{\prime}}\right)=\mathbf{f}^{n+1-\Theta}, \\
& \mathbf{u}^{n+1-\Theta}=\mathbf{g}^{n+1-\Theta} \quad \text { sobre } \Gamma, \\
& \frac{\mathbf{u}^{n+1}-\mathbf{u}^{n+1-\Theta}}{\Theta \Delta t}+A_{1}\left(\mathbf{u}^{n+1}\right)+A_{2}\left(\mathbf{u}^{n+1-\Theta}\right)=\mathbf{f}^{n+1} \\
& \mathbf{u}^{n+1}=\mathbf{g}^{n+1} \quad \text { sobre } \Gamma,
\end{aligned}
$$




\subsubsection{Aplicación a la simulación de un fluido newtoniano}

Al aplicar el esquema de descomposición de operadores (4.5) con la descomposición:

$$
\begin{aligned}
& A_{1}(\mathbf{u})=-\frac{\nu}{2} \Delta \mathbf{u}+(\mathbf{u} \cdot \nabla) \mathbf{u} \\
& A_{2}(\mathbf{u})=\left(\begin{array}{c}
-\frac{\nu}{2} \Delta \mathbf{u}+\nabla p \\
\nabla \cdot \mathbf{u}
\end{array}\right),
\end{aligned}
$$

con las condiciones iniciales (2.10) y con las condiciones de frontera (2.12), a la solución de las ecuaciones de Navier Stokes (2.3), las dos dificultades principales de tal problema (el término no lineal y la condición de incompresibilidad) se desacoplan y se obtienen los tres problemas siguientes:

Dado $\quad \mathbf{u}^{\mathbf{0}}=\mathbf{u}_{\mathbf{0}}$, para cada $n \geq 1$, con $\mathbf{u}^{n}$ conocido, calcular sucesivamente $\left\{\mathbf{u}^{n+\Theta}, p^{n+\Theta}\right\}, \mathbf{u}^{n+1-\Theta} y\left\{\mathbf{u}^{n+1}, p^{n+1}\right\}$ mediante:

$$
\begin{gathered}
\frac{\mathbf{u}^{n+\Theta}-\mathbf{u}^{n}}{\Theta \Delta t}-\alpha \vartheta \Delta \mathbf{u}^{n+\Theta}+\nabla p^{n+\Theta}=\mathbf{f}^{n+\Theta}+\beta \vartheta \Delta \mathbf{u}^{n}-\left(\mathbf{u}^{n} \cdot \nabla\right) \mathbf{u}^{n} \quad \text { en } \Omega, \\
\nabla \cdot \mathbf{u}^{n+\Theta}=0 \quad \text { en } \Omega, \\
\mathbf{u}^{n+\Theta}=\mathbf{g}^{n+\Theta} \quad \text { sobre } \Gamma_{\mathbf{0}} \\
\alpha \vartheta \frac{\partial \mathbf{u}^{n+\Theta}}{\partial n}-\mathbf{n} p^{n+\Theta}=\mathbf{g}^{n+\Theta}-\beta \vartheta \frac{\partial \mathbf{u}^{n}}{\partial n} \quad \text { sobre } \Gamma_{1}
\end{gathered}
$$




$$
\begin{gathered}
\frac{\mathbf{u}^{n+1-\Theta}-\mathbf{u}^{n+\Theta}}{(1-2 \Theta) \Delta t}-\beta \vartheta \Delta \mathbf{u}^{n+1-\Theta}+\left(\mathbf{u}^{n+1-\Theta} \cdot \nabla\right) \mathbf{u}^{n+1-\Theta}=\mathbf{f}^{n+1-\Theta}+ \\
a \vartheta \Delta \mathbf{u}^{n+\Theta}-\nabla p^{n+\Theta} \quad \text { en } \Omega \\
\mathbf{u}^{n+1-\Theta}=\mathbf{g}^{n+1-\Theta} \quad \text { sobre } \Gamma_{0} \\
\beta \vartheta \frac{\partial \mathbf{u}^{n+1-\Theta}}{\partial n}=\mathbf{g}_{1}^{n+1-\Theta}+\mathbf{n} p^{n+\Theta}-\alpha \vartheta \frac{\partial \mathbf{u}^{n+\Theta}}{\partial n} \quad \text { sobre } \Gamma_{1} \\
\frac{\mathbf{u}^{n+1}-\mathbf{u}^{n+1-\Theta}}{\Theta \Delta t}-\alpha \vartheta \Delta \mathbf{u}^{n+1}+\nabla p^{n+1}=\mathbf{f}^{n+1}+ \\
\beta \vartheta \Delta \mathbf{u}^{n+1-\Theta}-\left(\mathbf{u}^{n+1-\Theta} \cdot \nabla\right) \mathbf{u}^{n+1-\Theta} \quad \text { en } \Omega \\
\nabla \cdot \mathbf{u}^{n+1}=0 \quad \text { en } \Omega \\
\mathbf{u}^{n+1}=\mathbf{g}^{n+1} \quad \text { sobre } \Gamma_{0} \\
\alpha \vartheta \frac{\partial \mathbf{u}^{n+1}}{\partial n}-\mathbf{n} p^{n+1}=\mathbf{g}_{1}^{n+1}-\beta \vartheta \frac{\partial \mathbf{u}^{n+1-\Theta}}{\partial n} \quad \text { sobre } \Gamma_{1}
\end{gathered}
$$

Después de aplicar el método de punto fijo a los problemas elípticos (4.7) y (4.9), y el del gradiente conjugado al problema tipo Stokes (4.8), se formulan los problemas variacionales correspondientes de los dos problemas resultantes, y se discretizan los problemas variacionales así obtenidos por elemento finito (véanse las secciones (7) y (8)).

\subsection{Esquema de descomposición de operadores, de primer orden.}

Este método no requiere linealidad de los operadores, es sencillo de aplicar y permite descomponer los operadores en más de dos operadores. Su desventaja radica en que, por ser de primer orden en el tiempo, se obtienen buenos resultados solamente si el paso en el tiempo es relativamente pequeño. 


\subsubsection{Caso de descomposición en dos operadores}

Dado el problema de valor inicial (4.1), si $A$ se puede descomponer en la suma de dos operadores $A_{1}$ y $A_{2}$, tales que

$$
A=A_{1}+A_{2},
$$

entonces el método en cuestión proporciona un esquema de discretización en el tiempo, de dos pasos, que consiste en lo siguiente:

Dado $\quad \mathbf{u}^{0}=\mathbf{u}_{0}, \quad$ para cada $\quad n \geq 0, \quad$ con $\mathbf{u}^{n}$ conocido, calcular sucesivamente $\widetilde{\mathbf{u}}^{n+\frac{1}{2}}$ y $\widetilde{\mathbf{u}}^{n+1}$, mediante:

$$
\begin{gathered}
\frac{\widetilde{\mathbf{u}}^{n+\frac{1}{2}}-\mathbf{u}^{n}}{\Delta t}+A_{1}\left(\widetilde{\mathbf{u}}^{n+\frac{1}{2}}\right)=0, \\
\widetilde{\mathbf{u}}^{n+\frac{1}{2}}=\mathbf{g}^{n+\frac{1}{2}} \quad \text { sobre } \Gamma \\
\frac{\mathbf{u}}{\Delta t}+A_{2}\left(\mathbf{u}^{n+1}\right)=f^{n+1}, \\
\widetilde{\mathbf{u}}^{n+1}=\mathbf{g}^{n+1} \quad \text { sobre } \Gamma,
\end{gathered}
$$

en donde $\Delta t$ (positivo) representa el paso de discretización del tiempo y $\widetilde{\mathbf{u}}^{n+\frac{1}{2}}$ representa un valor auxiliar de $\mathbf{u}$ en un momento intermedio entre $n \Delta t \mathbf{y}$ $(n+1) \Delta t$, no perteneciente a la malla.

\subsubsection{Caso de descomposición en tres operadores}

Dado el problema de valor inicial (4.1), si $A$ se puede descomponer en la suma de tres operadores $A_{1}, A_{2}$ y $A_{3}$, tales que

$$
A=A_{1}+A_{2}+A_{3}
$$


entonces el método en cuestión proporciona un esquema de discretización en el tiempo, de tres pasos, que consiste en lo siguiente:

Dado $\quad \mathbf{u}^{0}=\mathbf{u}_{0}$, para cada $n \geq 0, \quad$ con $\mathbf{u}^{n}$ conocido, calcular sucesivamente $\widetilde{\mathbf{u}}^{n+\frac{1}{3}}, \widetilde{\mathbf{u}}^{n+\frac{2}{3}}$ y $\mathbf{u}^{n+1}$, mediante:

$$
\begin{gathered}
\frac{\widetilde{\mathbf{u}}^{n+\frac{1}{3}}-\mathbf{u}^{n}}{\Delta t}+A_{1}\left(\widetilde{\mathbf{u}}^{n+\frac{1}{3}}\right)=0, \\
\widetilde{\mathbf{u}}^{n+\frac{1}{3}}=\mathbf{g}^{n+\frac{1}{3}} \quad \text { sobre } \Gamma, \\
\frac{\widetilde{\mathbf{u}}^{n+\frac{2}{3}}-\widetilde{\mathbf{u}}^{n+\frac{1}{3}}}{\Delta t}+A_{2}\left(\widetilde{\mathbf{u}}^{n+\frac{2}{3}}\right)=0, \\
\frac{\widetilde{\mathbf{u}}^{n+\frac{2}{3}}=\mathbf{g}^{n+\frac{2}{3}}}{} \quad \text { sobre } \Gamma, \\
\Delta t \\
\widetilde{\mathbf{u}}^{n+1}-\widetilde{\mathbf{u}}^{n+\frac{2}{3}}+A_{\mathbf{3}}\left(\mathbf{u}^{n+1}\right)=\mathbf{f}^{n+1}, \\
\widetilde{\mathbf{u}}^{n+1}=\mathbf{g}^{n+1} \quad \text { sobre } \Gamma,
\end{gathered}
$$

en donde $\Delta t$ (positivo) representa el paso de discretización del tiempo y $\widetilde{\mathbf{u}}^{n+\frac{1}{3}}$ y $\widetilde{\mathbf{u}}^{n+\frac{2}{3}}$ representan valores auxiliares de $\mathbf{u}$ en momentos intermedios entre $n \Delta t$ y $(n+1) \Delta t$, no pertenecientes a la malla.

\subsubsection{Determinación del orden del método}

La solución general exacta de $(4.1)_{1}$ depende de la forma del operador $A$; sin embargo, integrando esa ecuación con respecto al tiempo, en el caso homogéneo, y utilizando el operador de $H$ en $H$ definido mediante la siguiente expresión:

$$
F=\exp (-t A)=I-t A+\frac{1}{2} t^{2} A^{2}+\cdots
$$


se obtiene

$$
\begin{gathered}
\mathbf{u}(\mathbf{x}, t)=\exp [-t A] \mathbf{u}(\mathbf{x}, 0)= \\
=\left[I-t A+\frac{1}{2} t^{2} A^{2}-\frac{1}{6} t^{3} A^{3}+\cdots\right] \mathbf{u}(\mathbf{x}, 0)
\end{gathered}
$$

ya que (recuerdese que se supuso que $\mathrm{f} \equiv 0$ y que $A$ es un operador formado solamente por operadores de derivación espacial, véase (4.1)):

$$
\begin{aligned}
\frac{\partial \mathbf{u}(\mathbf{x}, t)}{\partial t} & =-A[\mathbf{u}(\mathbf{x}, 0)]+t A^{2}[\mathbf{u}(\mathbf{x}, 0)]-\frac{1}{2} t^{2} A^{3}[\mathbf{u}(\mathbf{x}, 0)]+\cdots= \\
& =-A\left[\mathbf{u}(\mathbf{x}, 0)-t A[\mathbf{u}(\mathbf{x}, 0)]+\frac{1}{2} t^{2} A^{2}[\mathbf{u}(\mathbf{x}, 0)]-\cdots\right]= \\
& =-A\left[I-t A+\frac{1}{2} t^{2} A^{2}-\cdots\right] \mathbf{u}(\mathbf{x}, 0)= \\
& =-A \exp [-t A] \mathbf{u}(\mathbf{x}, 0)=-A[\mathbf{u}(\mathbf{x}, t)]
\end{aligned}
$$

Lo cual implica que $\mathbf{u}(\mathrm{x}, t)$ es una solución de $(4.1)_{1}$. Por otro lado, de $(4.11)_{1}$ :

$$
\begin{gathered}
\widetilde{\mathbf{u}}^{n+\frac{1}{2}}-\mathbf{u}^{n}+\Delta t A_{1}\left(\widetilde{\mathbf{u}}^{n+\frac{1}{2}}\right)=0 \\
\left(I+\Delta t A_{1}\right) \widetilde{\mathbf{u}}^{n+\frac{1}{2}}=\mathbf{u}^{n}
\end{gathered}
$$

Análogamente, de $(4.11)_{2}$, en el caso homogéneo:

$$
\left(I+\Delta t A_{2}\right) \mathbf{u}^{n+1}=\widetilde{\mathbf{u}}^{n+\frac{1}{2}}
$$

Sustituyendo (4.17) en (4.16): 


$$
\left(I+\Delta t A_{1}\right) \widetilde{\mathbf{u}}^{n+\frac{1}{2}}=\left(I+\Delta t A_{1}\right)\left(I+\Delta t A_{2}\right) \mathbf{u}^{n+1}=\mathbf{u}^{n}
$$

De donde, multiplicando ambos lados por $\left(I+\Delta t A_{2}\right)^{-1}\left(I+\Delta t A_{1}\right)^{-1}$ :

$$
\mathbf{u}^{n+1}=\left(I+\Delta t A_{2}\right)^{-1}\left(I+\Delta t A_{1}\right)^{-1} \mathbf{u}^{n}
$$

Es decir, para el valor de la solución general aproximada de $(4.1)_{1}$ en $t^{n+1}$, en el caso homogéneo, se cumple lo siguiente:

$$
\begin{aligned}
\mathbf{u}_{a p r o x}^{n+1} & =\mathbf{u}_{\text {aprox }}[\mathbf{x},(n+1) \Delta t]= \\
& =\left(I+\Delta t A_{2}\right)^{-1}\left(I+\Delta t A_{1}\right)^{-1} \mathbf{u}_{\text {aprox }}^{n}= \\
& =\left(I+\Delta t A_{2}\right)^{-1}\left(I+\Delta t A_{1}\right)^{-1} \mathbf{u}_{\text {aprox }}(\mathbf{x}, n \Delta t)
\end{aligned}
$$

Trasladando el orígen del sistema de coordenadas temporales a $t^{n}=n \Delta t$, se obtiene:

$$
\begin{gathered}
t^{n}=n \Delta t=0, \quad t^{n+1}=(n+1) \Delta t=\Delta t \\
\mathbf{u}_{\text {aprox }}(\mathbf{x}, \Delta t)=\left(I+\Delta t A_{2}\right)^{-1}\left(I+\Delta t A_{1}\right)^{-1} \mathbf{u}_{\text {aprox }}(\mathbf{x}, 0)
\end{gathered}
$$

Si se considera que:

$$
(I \pm \Delta t A)^{-1}=\left[I \mp \Delta t A+(\Delta t)^{2} A^{2} \mp(\Delta l)^{3} A^{3} \cdots\right]
$$

lo cual se puede comprobar multiplicando ambos lados por $(I+\Delta t A)$, entonces el esarrollando en serie de Taylor de $\mathbf{u}_{a p r c x}(\mathbf{x}, \Delta t)$ se puede obtener a partir de (4.19) como sigue: 


$$
\begin{gathered}
\mathbf{u}_{\text {aprox }}(\mathbf{x}, \Delta t)= \\
=\left[I-\Delta t A_{2}+(\Delta t)^{2} A_{2}^{2}-\cdots\right]\left[I-\Delta t A_{1}+(\Delta t)^{2} A_{1}^{2}-\cdots\right] \mathbf{u}_{\text {aprox }}(\mathbf{x}, 0) \\
=\left[I-\left(A_{1}+A_{2}\right) \Delta t+(\Delta t)^{2}\left(A_{1}^{2}+A_{1} A_{2}+A_{2}^{2}\right)+\cdots\right] \mathbf{u}_{\text {aprox }}(\mathbf{x}, 0)= \\
=\left[I-\Delta t A+(\Delta t)^{2}\left(A_{1}^{2}+A_{1} A_{2}+A_{2}^{2}\right)+\cdots\right] \mathbf{u}_{\text {aprox }}(\mathbf{x}, 0)=
\end{gathered}
$$

Comparando esta expresión con (4.15), se concluye que el desarrollo de Maclaurin de la solución general aproximada de (4.1) obtenida con el método en cuestión, coincide hasta el término de primer orden (segundo de la serie), con el desarrollo de Taylor de la solución general exacta y que, por ello, este método es tiene una precisión de primer orden.

\subsubsection{Aplicación a la simulación de un fluido newtoniano}

Al aplicar el esquema de descomposición de operadores de primer orden (4.10)-(4.11) con la descomposición:

$$
\begin{aligned}
& A_{1}(\mathbf{u})=-\frac{\nu}{2} \Delta \mathbf{u}+(\mathbf{u} \cdot \nabla) \mathbf{u} \\
& A_{2}(\mathbf{u})=\left(\begin{array}{c}
-\frac{\nu}{2} \Delta \mathbf{u}+\nabla p \\
\nabla \cdot \mathbf{u}
\end{array}\right),
\end{aligned}
$$

con las condiciones iniciales (2.10) y con las condiciones de frontera (2.11), a la solución de las ecuaciones de Navier Stokes (2.3), las dos dificultades principales de tal problema (el término no lineal y la condición de incompresibilidad) se desacoplan y se obtienen los dos problemas siguientes:

Dado $\mathbf{u}^{0}=\mathbf{u}_{0}$, para cada $n \geq 0$, con $\mathbf{u}^{n}$ conocido, calcular sucesivamente $\widetilde{\mathbf{u}}^{n+\frac{1}{2}} y\left\{\mathbf{u}^{n+1}, p^{n+1}\right\}$ mediante: 


$$
\begin{aligned}
& \frac{\widetilde{\mathbf{u}}^{n+\frac{1}{2}}-\mathbf{u}^{n}}{\Delta t}-\frac{\nu}{2} \Delta \widetilde{\mathbf{u}}^{n+\frac{1}{2}}+\left(\widetilde{\mathbf{u}}^{n+\frac{1}{2}} \cdot \nabla\right) \widetilde{\mathbf{u}}^{n+\frac{1}{2}}=0 \quad \text { en } \Omega, \\
& \widetilde{\mathbf{u}}^{n+\frac{1}{2}}=\mathbf{g}^{n+\frac{1}{2}} \quad \text { sobre } \Gamma \\
& \mathbf{y} \\
& \frac{\mathbf{u}^{n+1}-\widetilde{\mathbf{u}}^{n+\frac{1}{2}}}{\Delta t}-\frac{\nu}{2} \Delta \mathbf{u}^{n+1}+\nabla p^{n+1}=\mathbf{f}^{n+1} \quad \text { en } \Omega \\
& \nabla \cdot \mathbf{u}^{n+1}=0 \quad \text { en } \Omega, \\
& \mathbf{u}^{n+1}=\mathbf{g}^{n+1} \quad \text { sobre } \Gamma .
\end{aligned}
$$

Después de aplicar el método de punto fijo al problema elíptico (4.21) y el del gradiente conjugado al problema tipo Stokes (4.22), se formulan los problemas variacionales correspondientes de los dos problemas resultantes, y se discretizan los problemas variacionales así obtenidos por elemento finito (véanse las secciones (7) y (8)).

\subsection{Método de Dyakonov}

El método de Dyakonov consiste en aplicar dos veces el esquema de descomposición de operadores de primer orden descrito en la sección (4.3), como se muestra en los siguientes incisos. Este método se puede aplicar a cualquier cantidad finita de operadores y es de segundo orden. 


\subsubsection{Caso de descomposición en dos operadores}

Dado el problema de valor inicial (4.1), si $A$ se puede descomponer en dos operadores $A_{1}$ y $A_{2}$ que satisfagan (4.2), entonces se puede obtener un esquema de discretización de cuatro pasos, de la variable temporal de ese problema, como sigue:

Dado $\quad \mathbf{u}^{0}=\mathbf{u}_{0}$, para cada $n \geq 0, \quad$ con $\mathbf{u}^{n}$ conocido, calcular sucesivamente $\widetilde{\mathbf{u}}^{n+\frac{1}{4}}, \widetilde{\mathbf{u}}^{n+\frac{1}{2}}, \widetilde{\mathbf{u}}^{n+\frac{3}{4}} \mathbf{y} \mathbf{u}^{n+1}$, mediante:

* Primer ciclo (explícito en la incógnita)

$$
\begin{gathered}
\frac{\widetilde{\mathbf{u}}^{n+\frac{1}{4}}-\mathbf{u}^{n}}{\Delta t}+\frac{1}{2} A_{1}\left(\mathbf{u}^{n}\right)=0, \\
\widetilde{\mathbf{u}}^{n+\frac{1}{4}}=\mathbf{g}^{n+\frac{1}{4}} \quad \text { sobre } \Gamma, \\
\frac{\widetilde{\mathbf{u}}^{n+\frac{1}{2}}-\widetilde{\mathbf{u}}^{n+\frac{1}{4}}}{\Delta t}+\frac{1}{2} A_{2}\left(\widetilde{\mathbf{u}}^{n+\frac{1}{4}}\right)=\mathbf{f}^{n+\frac{1}{4}}, \\
\widetilde{\mathbf{u}}^{n+\frac{1}{2}}=\mathbf{g}^{n+\frac{1}{2}} \quad \text { sobre } \Gamma,
\end{gathered}
$$

* Segundo ciclo (implícito en la incógnita)

$$
\begin{gathered}
\frac{\widetilde{\mathbf{u}}^{n+\frac{3}{4}}-\widetilde{\mathbf{u}}^{n+\frac{1}{2}}}{\Delta t}+\frac{1}{2} A_{2}\left(\widetilde{\mathbf{u}}^{n+\frac{3}{4}}\right)=0, \\
\widetilde{\mathbf{u}}^{n+\frac{3}{4}}=\mathbf{g}^{n+\frac{3}{4}} \quad \text { sobre } \Gamma, \\
\frac{\mathbf{u}^{n+1}-\widetilde{\mathbf{u}}^{n+\frac{3}{4}}}{\Delta t}+\frac{1}{2} A_{1}\left(\mathbf{u}^{n+1}\right)=\mathbf{f}^{n+1} . \\
\widetilde{\mathbf{u}}^{n+1}=\mathbf{g}^{n+1} \quad \text { sobre } \Gamma,
\end{gathered}
$$

en donde $\Delta t$ (positivo) representa el paso de discretización del tiempo y $\widetilde{\mathbf{u}}^{n+\frac{1}{4}}, \widetilde{\mathbf{u}}^{n+\frac{1}{2}}$ y $\widetilde{\mathbf{u}}^{n+\frac{3}{4}}$, representan valores auxiliares de $\mathbf{u}$ en momentos intermedios entre $n \Delta t$ y $(n+1) \Delta t$, no pertenecientes a la malla. 


\subsubsection{Caso de descomposición en tres operadores}

Dado el problema de valor inicial (4.1), si $A$ se puede descomponer en tres operadores $A_{1}, A_{2}$ y $A_{3}$, cuya suma sea igual a $A$, entonces se puede obtener un esquema de discretización de seis pasos, de la variable temporal de ese problema, como sigue:

Dado $\mathbf{u}^{\mathbf{0}}=\mathbf{u}_{\mathbf{0}}$, para cada $n \geq 0$, con $\mathbf{u}^{n}$ conocido, calcular sucesivamente $\widetilde{\mathbf{u}}^{n+\frac{1}{6}}, \widetilde{\mathbf{u}}^{n+\frac{1}{3}}, \widetilde{\mathbf{u}}^{n+\frac{1}{2}}, \widetilde{\mathbf{u}}^{n+\frac{2}{3}}, \widetilde{\mathbf{u}}^{n+\frac{5}{6}}$ y $\mathbf{u}^{n+1}$, mediante:

* Primer ciclo (explícito en la inógnita)

$$
\begin{gathered}
\frac{\widetilde{\mathbf{u}}^{n+\frac{1}{6}}-\mathbf{u}^{n}}{\Delta t}+\frac{1}{2} A_{1}\left(\mathbf{u}^{n}\right)=0, \\
\widetilde{\mathbf{u}}^{n+\frac{1}{6}}=\mathbf{g}^{n+\frac{1}{6}} \quad \text { sobre } \Gamma, \\
\frac{\widetilde{\mathbf{u}}^{n+\frac{1}{3}}-\widetilde{\mathbf{u}}^{n+\frac{1}{6}}}{\Delta t}+\frac{1}{2} A_{2}\left(\widetilde{\mathbf{u}}^{n+\frac{1}{6}}\right)=0, \\
\widetilde{\mathbf{u}}^{n+\frac{1}{3}}=\mathbf{g}^{n+\frac{1}{3}} \quad \text { sobre } \Gamma, \\
\frac{\widetilde{\mathbf{u}}^{n+\frac{1}{2}}-\widetilde{\mathbf{u}}^{n+\frac{1}{3}}}{\Delta t}+\frac{1}{2} A_{3}\left(\widetilde{\mathbf{u}}^{n+\frac{1}{3}}\right)=\mathbf{f}^{n+\frac{1}{3}}, \\
\widetilde{\mathbf{u}}^{n+\frac{1}{2}}=\mathbf{g}^{n+\frac{1}{2}} \quad \text { sobre } \Gamma,
\end{gathered}
$$

* Segundo ciclo (implícito en la incógnita)

$$
\begin{gathered}
\frac{\widetilde{\mathbf{u}}^{n+\frac{2}{3}}-\widetilde{\mathbf{u}}^{n+\frac{1}{2}}}{\Delta t}+\frac{1}{2} A_{3}\left(\widetilde{\mathbf{u}}^{n+\frac{2}{3}}\right)=0 \\
\widetilde{\mathbf{u}}^{n+\frac{2}{3}}=\mathbf{g}^{n+\frac{2}{3}} \quad \text { sobre } \Gamma,
\end{gathered}
$$




$$
\begin{gathered}
\frac{\widetilde{\mathbf{u}}^{n+\frac{5}{6}}-\widetilde{\mathbf{u}}^{n+\frac{2}{3}}}{\Delta t}+\frac{1}{2} A_{2}\left(\widetilde{\mathbf{u}}^{n+\frac{5}{6}}\right)=0 \\
\widetilde{\mathbf{u}}^{n+\frac{5}{6}}=\mathbf{g}^{n+\frac{5}{6}} \quad \text { sobre } \Gamma, \\
\frac{\mathbf{u}^{n+1}-\widetilde{\mathbf{u}}^{n+\frac{5}{6}}}{\Delta t}+\frac{1}{2} A_{1}\left(\mathbf{u}^{n+1}\right)=\mathbf{f}^{n+1} \\
\widetilde{\mathbf{u}}^{n+1}=\mathbf{g}^{n+1} \quad \text { sobre } \Gamma,
\end{gathered}
$$

en donde $\Delta t$ (positivo) representa el paso de discretización del tiempo y $\widetilde{\mathbf{u}}^{n+\frac{1}{6}}, \widetilde{\mathbf{u}}^{n+\frac{1}{3}}, \widetilde{\mathbf{u}}^{n+\frac{1}{2}}, \widetilde{\mathbf{u}}^{n+\frac{2}{3}}$ y $\widetilde{\mathbf{u}}^{n+\frac{3}{6}}$, representan valores auxiliares de $\mathbf{u}$ en momentos intermedios entre $n \Delta t$ y $(n+1) \Delta t$, no pertenecientes a la malla.

\subsubsection{Determinación del orden del método}

Los cálculos se hacen para el caśo de descomposición en dos operadores. De $(4.23)_{1}$ :

$$
\begin{gathered}
\widetilde{\mathbf{u}}^{n+\frac{1}{4}}-\mathbf{u}^{n}+\frac{1}{2} \Delta t A_{1}\left(\mathbf{u}^{n}\right)=0 \\
\widetilde{\mathbf{u}}^{n+\frac{1}{4}}=\left(I-\frac{1}{2} \Delta t A_{1}\right) \mathbf{u}^{n}
\end{gathered}
$$

Análogamente, de $(4.23)_{2},(4.24)_{1} \mathbf{y}(4.24)_{2}$, en el caso homogéneo, se obtiene respectivamente lo siguiente:

$$
\begin{aligned}
& \widetilde{\mathbf{u}}^{n+\frac{1}{2}}=\left(I-\frac{1}{2} \Delta t A_{2}\right) \mathbf{u}^{n+\frac{1}{4}} \\
& \widetilde{\mathbf{u}}^{n+\frac{1}{2}}=\left(I+\frac{1}{2} \Delta t A_{2}\right) \widetilde{\mathbf{u}}^{n+\frac{3}{4}} \\
& \widetilde{\mathbf{u}}^{n+\frac{3}{4}}=\left(I+\frac{1}{2} \Delta t A_{1}\right) \mathbf{u}^{n+1}
\end{aligned}
$$


Sustituyendo (4.27) en (4.28):

$$
\widetilde{\mathbf{u}}^{n+\frac{1}{2}}=\left(I-\frac{1}{2} \Delta t A_{2}\right)\left(I-\frac{1}{2} \Delta t A_{1}\right) \mathbf{u}^{n}
$$

Multiplicando ambos lados de (4.30) por $\left(I+\frac{1}{2} \Delta t A_{2}\right)$ y tomando en cuenta la ecuación (4.29):

$$
\left(I+\frac{1}{2} \Delta t A_{2}\right) \widetilde{\mathbf{u}}^{n+\frac{3}{4}}=\left(I+\frac{1}{2} \Delta t A_{2}\right)\left(I+\frac{1}{2} \Delta t A_{1}\right) \mathbf{u}^{n+1}=\widetilde{\mathbf{u}}^{n+\frac{1}{2}}
$$

Despejando $\mathbf{u}^{n+1}$ :

$$
\mathbf{u}^{n+1}=\left(I+\frac{1}{2} \Delta t A_{1}\right)^{-1}\left(I+\frac{1}{2} \Delta t A_{2}\right)^{-1} \widetilde{\mathbf{u}}^{n+\frac{1}{2}}
$$

Sustituyendo (4.31) en (4.32)

$$
\begin{gathered}
\mathbf{u}^{n+1}= \\
\left(I+\frac{1}{2} \Delta t A_{1}\right)^{-1}\left(I+\frac{1}{2} \Delta t A_{2}\right)^{-1}\left(I-\frac{1}{2} \Delta t A_{2}\right)\left(I-\frac{1}{2} \Delta t A_{1}\right) \mathbf{u}^{n}
\end{gathered}
$$

Trasladando el origen del sistema de coordenadas temporales a $t^{n}=n \Delta t$, en forma similar a como se hizo en el inciso (4.3.3), se obtiene:

$$
\begin{gathered}
\mathbf{u}_{\text {aprox }}(\mathbf{x}, \Delta t)= \\
\left(I+\frac{1}{2} \Delta t A_{1}\right)^{-1}\left(I+\frac{1}{2} \Delta t A_{2}\right)^{-1}\left(I-\frac{1}{2} \Delta t A_{2}\right)\left(I-\frac{1}{2} \Delta t A_{1}\right) \mathbf{u}_{\text {aprox }}(\mathbf{x}, 0)
\end{gathered}
$$


Desarrollando en serie de Taylor y tomando en cuenta que (véase (4.20)):

$$
\left(I \pm \frac{1}{2} \Delta t A_{i}\right)^{-1}=\left(I \mp \frac{1}{2} \Delta t A_{i}+\frac{1}{2}(\Delta t)^{2} A_{i}^{2} \mp \cdots\right),
$$

se obtiene la expresión signiente:

$$
\begin{gathered}
\mathbf{u}_{\text {aprox }}(\mathbf{x}, \Delta t)= \\
\left(I+\frac{1}{2} \Delta t A_{1}\right)^{-1}\left(I+\frac{1}{2} \Delta t A_{2}\right)^{-1}\left(I-\frac{1}{2} \Delta t A_{2}\right)\left(I-\frac{1}{2} \Delta t A_{1}\right) \mathbf{u}_{\text {aprox }}(\mathbf{x}, 0)= \\
{\left[I-\Delta t A_{1}-\Delta t A_{2}+2 \frac{1}{2}(\Delta t) A_{1} A_{2}+\frac{1}{2}(\Delta t)^{2} A_{1}^{2}+\frac{1}{2}(\Delta t)^{2} A_{1}^{2}+\ldots\right] \mathbf{u}_{\text {aprox }}(\mathbf{x}, 0)=} \\
=\left[I-\Delta t\left(A_{1}+A_{2}\right)+\frac{1}{2}(\Delta t)^{2}\left(A_{1}^{2}+2 A_{1}^{2} A_{2}^{2}+A_{2}^{2}\right)+\cdots\right] \mathbf{u}_{a p r o x}(\mathbf{x}, 0)= \\
=\left[I-\Delta t A+\frac{1}{2}(\Delta t)^{2} A^{2}+\cdots\right] \mathbf{u}_{\text {aprox }}(\mathbf{x}, 0)
\end{gathered}
$$

Es decir:

$$
\mathbf{u}_{a p r o x}(\mathbf{x}, \Delta t)=\left[I-\Delta t A+\frac{1}{2}(\Delta t)^{2} A^{2}+\cdots\right] \mathbf{u}_{a p r o x}(\mathbf{x}, 0)
$$

Comparando esta expresión con (4.15), se concluye que el desarrollo de Maclaurin de la solución general aproximada de (4.1) $)_{1}$ obtenida con el método en cuestión, coincide hasta el término de segundo orden (tercero de la serie) con el desarrollo de Maclaurin de la solución general exacta y que, por ello, este método tiene una precisión de segundo orden. 


\subsubsection{Aplicación a la simulación de un fluido newtoniano}

$\mathrm{Al}$ aplicar el esquema de descomposición de operadores (4.23)-(4.24) con la descomposición:

$$
\begin{aligned}
& A_{1}(\mathbf{u})=\left(\begin{array}{c}
-\frac{\nu}{2} \Delta \mathbf{u}+\nabla p \\
\nabla \cdot \mathbf{u}
\end{array}\right) \\
& A_{2}(\mathbf{u})=-\frac{\nu}{2} \Delta \mathbf{u}+(\mathbf{u} \cdot \nabla) \mathbf{u}
\end{aligned}
$$

con las condiciones de iniciales (2.10) y con las condiciones de frontera (2.11), a la solución de las ecuaciones de Navier Stokes (2.3), las dos dificultades principales de tal problema (el término no lineal y la condición de incompresibilidad) se desacoplan y se obtienen los dos problemas siguientes:

Dado $\quad \mathbf{u}^{0}=\mathbf{u}_{0}$, para cada $n \geq 0, \quad$ con $\mathbf{u}^{n}$ conocido, calcular sucesivamente $\widetilde{\mathbf{u}}^{n+\frac{1}{4}}, \widetilde{\mathbf{u}}^{n+\frac{1}{2}}, \widetilde{\mathbf{u}}^{n+\frac{3}{4}}$ y $\left\{\mathbf{u}^{n+1}, p^{n+1}\right\}$, mediante:

* Primer ciclo (explícito en la incógnita)

$$
\begin{gathered}
\frac{\widetilde{\mathbf{u}}^{n+\frac{1}{4}}-\mathbf{u}^{n}}{\Delta t}-\frac{\nu}{4} \Delta \mathbf{u}^{n}+\frac{1}{2} \nabla p^{n}=0 \\
\nabla \cdot \mathbf{u}^{n}=0 \\
\widetilde{\mathbf{u}}^{n+\frac{1}{4}}=\mathbf{g}^{n+\frac{1}{4}} \quad \text { sobre } \Gamma \\
\mathbf{y} \\
\frac{\widetilde{\mathbf{u}}^{n+\frac{1}{2}}-\mathbf{u}^{n+\frac{1}{4}}}{\Delta t}-\frac{\nu}{4} \Delta \mathbf{u}^{n+\frac{1}{4}}+\frac{1}{2}\left(\mathbf{u}^{n+\frac{1}{4}} \cdot \nabla\right) \mathbf{u}^{n+\frac{1}{4}}=0 \quad \text { en } \Omega, \\
\widetilde{\mathbf{u}}^{n+\frac{1}{2}}=\mathbf{g}^{n+\frac{1}{2}} \quad \text { sobre } \Gamma,
\end{gathered}
$$


* Segundo ciclo (implícito en la incógnita)

$$
\begin{gathered}
\frac{\widetilde{\mathbf{u}}^{n+\frac{3}{4}}-\widetilde{\mathbf{u}}^{n+\frac{1}{2}}}{\Delta t}-\frac{\nu}{4} \Delta \widetilde{\mathbf{u}}^{n+\frac{3}{4}}+\frac{1}{2}\left(\widetilde{\mathbf{u}}^{n+\frac{3}{4}} \cdot \nabla\right) \widetilde{\mathbf{u}}^{n+\frac{3}{4}}=0 \quad \text { en } \Omega \\
\widetilde{\mathbf{u}}^{n+\frac{3}{4}}=\mathbf{g}^{n+\frac{3}{4}} \quad \text { sobre } \Gamma . \\
\mathbf{y} \quad \text { en } \Omega, \\
\frac{\mathbf{u}^{n+1}-\widetilde{\mathbf{u}}^{n+\frac{3}{4}}}{\Delta t}-\frac{\nu}{4} \Delta \mathbf{u}^{n+1}+\frac{1}{2} \nabla p^{n+1}=\mathbf{f}^{n+1} \quad \mathbf{u}^{n+1}=0 \\
\mathbf{u}^{n+1}=\mathbf{g}^{n+1} \quad \text { sobre } \Gamma .
\end{gathered}
$$

Después de resolver los problemas (4.36) y (4.37) como problemas explicitos que son, y después de aplicar el método de punto fijo al problema elíptico (4.38) y el del gradiente conjugado al problema tipo Stokes (4.39), se formulan los problemas variacionales que corresponden a cada uno de los dos problemas que resultan de la aplicación de esos métodos, y se discretizan, mediante elemento finito, los problemas variacionales así obtenidos (véanse secciones (7) y (8)).

\section{Solución del problema elíptico, no lineal, de advección-difusión, por el método itera- tivo de punto fijo.}

Para resolver las ecuaciones de Navier-Stokes con condiciones de frontera tipo Dirichlet por los métodos de descomposición de operadores, por cada subintervalo del tiempo $[n \Delta t,(n+1) \Delta t]$, se tiene que resolver uno o varios subroblemas espaciales no lineales de tipo elíptico (problemas de advecciondifusion) de la siguiente forma (véase el subproblema (4.21)): 


$$
\begin{gathered}
a \mathbf{u}-\mu \Delta \mathbf{u}+(\mathbf{u} \cdot \nabla) \mathbf{u}=\mathbf{f} \quad \text { en } \Omega \\
\mathbf{u}=\mathbf{g} \quad \text { sobre } \Gamma,
\end{gathered}
$$

en donde ([9], pags. 18-31), en el caso del método de primer orden y descomposición en dos operadores, $a=1 / \Delta t, \mu=\nu / 2, \quad \mathbf{u}=\mathbf{u}^{n+\frac{1}{2}}, \quad \mathbf{f}=\mathbf{u}^{n} / \Delta t \quad \mathbf{y}$ $\mathbf{g}=\mathbf{g}^{n+\frac{1}{2}}$, la función $\mathbf{u}=\mathbf{u}(\mathbf{x}, \mathbf{y})=\left(u_{1}(x, y), u_{2}(x, y)\right)$ solo depende de las coordenadas espaciales $\mathrm{y}$ las funciones $\mathrm{f} \mathbf{y} \mathrm{g}$ son funciones dadas $\mathrm{y}$ definidas en $\Omega$ y $\Gamma$, respectivamente. Introduciendo

$$
\mathbf{R}(\mathbf{u})=(a I-\mu \Delta) \mathbf{u}+(\mathbf{u} \cdot \nabla) \mathbf{u}-\mathbf{f}
$$

y considerando que para la solución $\mathrm{x}^{*}$ de (5.1) se cumple

$$
\mathbf{R}\left(\mathbf{u}^{*}\right)=0,
$$

se infiere que se cumple también:

$$
(a I-\mu \Delta) \mathbf{u}^{*}=(a I-\mu \Delta) \mathbf{u}^{*}-\mathbf{R}\left(\mathbf{u}^{*}\right)
$$

o, multiplicando por $(a I-\mu \Delta)^{-1}$ :

$$
\mathbf{u}^{*}=\mathbf{u}^{*}-(a I-\mu \Delta)^{-1} \mathbf{R}\left(\mathbf{u}^{*}\right)
$$

lo cual implica que, en cada subintervalo del tiempo $[n \Delta t,(n+1) \Delta t],(5.1)$ se puede resolver mediante el siguiente algoritmo iterativo de punto fijo:

Dado $\mathbf{u}^{\mathbf{0}}=\mathbf{u}^{n}$, para $m \geq 0$ (se elige $m$ para no confundirla con el orden $n$ de la iteración temporal), resolver hasta convergencia

$$
\mathbf{u}^{m+1}=\mathbf{u}^{m}-\rho(a \mathbf{I}-\mu \Delta)^{-1} \mathbf{R}\left(\mathbf{u}^{m}\right) \quad \text { en } \quad \Omega
$$




$$
\mathbf{u}^{m+1}=\mathbf{g}^{m+1} \quad \text { sobre } \Gamma
$$

luego se toma $\mathbf{u}^{(n+1 / 2, m+1)}=\mathbf{u}^{m+1}$ (véase 4.21 ), se hace $m=m+1$ y se regresa a $(5.6)_{1}$.

El problema (5.6) se resuelve mediante el siguiente esquema discreto de elemento finito ([10], pags. 9-10), el cual se obtuvo considerando que (5.6) se puede escribir como sigue:

$$
(a \mathbf{I}-\mu \Delta)\left(\mathbf{u}^{m+1}-\mathbf{u}^{m}\right)=(a \mathbf{I}-\mu \Delta)\left(\mathbf{w}^{m}\right)=-\rho \mathbf{R}\left(\mathbf{u}^{m}\right)
$$

y permite determinar, en el caso del método de primer orden y descomposición en dos operadores, para el valor $\left(n+\frac{1}{2}\right) \Delta t$ del tiempo y para cada valor de $m$, los valores de la $(m+1)$-ésima aproximación de la función $\mathbf{u}=\mathbf{u}\left[x, y,\left(n+\frac{1}{2}\right) \Delta t\right]$, en los puntos de la malla espacial, a partir de los valores de la m-ésima aproximación de esa función ([10], pags. 9-10):

Dado $\mathbf{u}_{h}^{\mathbf{0}} \in \mathbf{V}_{g h}$, para $m \geq 0$, resolver hasta convergencia:

Encontrar $\mathbf{w}_{h}^{m} \in \mathbf{V}_{0 h}$, tal que $\forall \mathbf{z}_{h} \in V_{0 h}$,

$$
\begin{gathered}
a \int_{\Omega} \mathbf{w}_{h}^{m} \cdot \mathbf{z}_{h} d x+\mu \int_{\Omega} \nabla \mathbf{w}_{h}^{m} \cdot \nabla \mathbf{z}_{h} d x=-\mathbf{R}_{h}\left(\mathbf{u}_{h}^{m}\right), \\
\mathbf{u}_{h}^{m+1}=\mathbf{u}_{h}^{m}-\rho \mathbf{w}_{h}^{m}
\end{gathered}
$$

en donde:

$$
\begin{gathered}
\mathbf{R}_{h}\left(\mathbf{u}_{h}^{m}\right)= \\
=a \int_{\Omega} \mathbf{u}_{h}^{m} \cdot \mathbf{z}_{h} d x+\mu \int_{\Omega} \nabla \mathbf{u}_{h}^{m} \cdot \nabla \mathbf{z}_{h} d x+\int_{\Omega}\left(\mathbf{u}_{h}^{m} \cdot \nabla\right) \mathbf{u}_{h}^{m} \cdot \mathbf{z}_{h} d x-\int_{\Omega} \mathbf{f}_{h} \cdot \mathbf{z}_{h} d x(5.8)_{1}
\end{gathered}
$$


y (véase la sección $(5)$ ): $\mathcal{T}_{h}$ representa la triangulación de $\Omega$ utilizada para aproximar la presión; $h$ representa la longitud del mayor lado de los triángulos de $\mathcal{T}_{h} ; \mathcal{P}_{k}$ es el espacio de los polinomios de grado menor o igual a $k$;

$$
H_{h}^{1}=\left\{\psi_{h} \in C^{0}(\bar{\Omega})\left|\psi_{h}\right|_{T} \in \mathcal{P}_{1}, \forall T \in \mathcal{T}_{h}\right\}
$$

es el espacio de funciones utilizado para aproximar la presión; $\widetilde{\mathcal{T}}_{h}$ se define como la triangulación de $\Omega$ que se obtiene, a partir de $\mathcal{T}_{h}$, uniendo los puntos medios de cada uno de sus triángulos;

$$
\mathbf{V}_{\boldsymbol{h}}=\left\{\mathbf{v}_{\boldsymbol{h}}\left|\mathbf{v}_{\boldsymbol{h}} \in C^{\mathbf{0}}(\bar{\Omega}) \times C^{\mathbf{0}}(\bar{\Omega}), \mathbf{v}_{\boldsymbol{h}}\right|_{T} \in \mathcal{P}_{1} \times \mathcal{P}_{1}, \forall T \in \tilde{\mathcal{T}}_{h}\right\}
$$

es el espacio de funciones utilizadas para aproximar la velocidad (lo cual implica que la malla de la velocidad es dos veces más fina que la correspondiente a la presión);

$$
\begin{aligned}
& \mathbf{V}_{g h}=\left\{\mathbf{v}_{h} \in \mathbf{V}_{h}=\mathbf{g}_{h} \text { sobre } \Gamma\right\} \\
\dot{\mathbf{y}} \quad & \mathbf{V}_{\mathbf{O} h}=\left\{\mathbf{v}_{h} \in \mathbf{V}_{h} \mid \mathbf{v}_{h}=\mathbf{0} \text { sobre } \Gamma\right\} .
\end{aligned}
$$

\section{Solución del problema tipo Stokes por el método del gradiente conjugado}

\subsection{Algoritmos básicos}

Para resolver las ecuaciones de Navier-Stokes con condiciones de frontera tipo Dirichlet por los métodos de descomposición de operadores, por cada subintervalo del tiempo $[n \Delta t,(n+1) \Delta t]$, se tiene que resolver uno o varios subroblemas espaciales tipo Stokes [problema (2.1) sin el término no lineal o término de advección ([9], pags. 32-50), el cual modela flujos de fluidos de gran viscocidad] de la siguiente forma (véase el subproblema (4.22)): 


$$
\begin{gathered}
a \mathbf{u}-\mu \Delta \mathbf{u}+\nabla p=\mathbf{f} \quad \text { en } \Omega \\
\nabla \cdot \mathbf{u}=\mathbf{0} \quad \text { en } \Omega \\
\mathbf{u}=\mathbf{g} \quad \text { sobre } \Gamma
\end{gathered}
$$

en donde, en el caso del método de primer orden y descomposición en dos operadores, $\quad a=\frac{1}{\Delta t}, \quad \mu=\frac{\nu}{2}, \quad \mathbf{u}=\mathbf{u}^{n+1}, \quad \mathbf{f}=\mathbf{f}^{n+1}+\frac{\mathbf{u}^{n}}{\Delta t}, \quad \mathbf{g}=\mathbf{g}^{n+1}$, y f y $g$ representan funciones dadas. Se puede demostrar que si $f$ y $g$ son suficientemente suaves, entonces ese problema tiene solución única en $\mathrm{V}_{g} \times H^{1}(\Omega)$.

Ese tipo de problemas se puede resolver discretizándolos directamente mediante elemento finito y resolviendo los sistemas de ecuaciones resultantes por el método del gradiente conjugado ([5], pags. 241-261), pero es más conveniente usar el siguiente algoritmo iterativo:

Dado $\quad p^{0} \in L^{2}(\Omega)$, entonces, para $m \geq 0$ (se elige $m$ para no confundirla con el orden $n$ de la iteración temporal), calcular $\mathbf{u}^{m}$ y $p^{m+1}$, a partir de $p^{m}$, como sigue:

$$
\begin{gathered}
a \mathbf{u}^{m}-\mu \Delta \mathbf{u}^{m}=\mathbf{f}-\nabla p^{m} \quad \text { en } \Omega, \\
\mathbf{u}^{m}=\mathbf{g} \quad \text { sobre } \Gamma, \\
p^{m+1}=p^{m}-\rho \nabla \cdot \mathbf{u}^{m}
\end{gathered}
$$

Fste algoritmo converge en $\left(H^{1}(\Omega)\right)^{N} \times L^{2}(\Omega)$ para $0<\rho<2 \frac{\mu}{N} \quad y$, aunque, en general es lento, trabaja bien con números de Reynolds moderados. Sin embargo, sus propiedades de convergencia se pueden mejorar usando el signiente algoritmo de gradiente conjugado: 
* Paso cero (inicialización)

Dado $\quad p^{0} \in L_{0}^{2}(\Omega), \quad$ calcular:

$$
\begin{gathered}
a \mathbf{u}^{\mathbf{0}}-\mu \Delta \mathbf{u}^{\mathbf{0}}=\mathbf{f}-\nabla p^{\mathbf{0}} \quad \text { en } \Omega, \\
\mathbf{u}^{\mathbf{0}}=\mathbf{g} \quad \text { sobre } \Gamma,
\end{gathered}
$$

A partir del valor de $\mathbf{u}^{0}$ así obtenido, definir (solamente en la primera iteración):

$$
g^{0}=\nabla \cdot \mathbf{u}^{0} \quad \text { y } \quad w^{0}=g^{0}
$$

Luego, para $m \geq 0$, dados $\left\{p^{m}, \mathbf{u}^{m}, g^{m}, w^{m}\right\}$, calcular $\left\{\overline{\mathbf{u}}^{m}, \bar{g}^{m}, p^{m+1}, \mathbf{u}^{m+1}, g^{m+1}, w^{m+1}\right\}$ como sigue:

\section{* Primer paso (descenso)}

Resolver:

$$
\begin{gathered}
a \overline{\mathbf{u}}^{m}-\mu \Delta \overline{\mathbf{u}}^{m}=-\nabla w^{m} \quad \text { en } \Omega \\
\overline{\mathbf{u}}^{m}=0 \quad \text { sobre } \Gamma
\end{gathered}
$$

considerar:

$$
\bar{g}^{m}=\nabla \cdot \overline{\mathbf{u}}^{m}
$$

y calcular:

$$
\rho_{m}=\frac{\left\|g^{m}\right\|_{L^{2}(\Omega)}^{2}}{\int_{\Omega}\left(a\left|\overline{\mathbf{u}}^{m}\right|^{2}+\mu\left|\nabla \overline{\mathbf{u}}^{m}\right|^{2}\right) d x}
$$




$$
\begin{aligned}
& p^{m+1}=p^{m}-\rho_{m} w^{m}, \\
& \mathbf{u}^{m+1}=\mathbf{u}^{m}-\rho_{m} \overline{\mathbf{u}}^{m}, \\
& g^{m+1}=g^{m}-\rho_{m} \bar{g}^{m} .
\end{aligned}
$$

Si $\frac{\left\|g^{m+1}\right\|_{L^{2}(\Omega)}}{\left\|g^{0}\right\|_{L^{2}(\Omega)}} \leq \epsilon$, entonces tomar $\mathbf{u}=\mathbf{u}^{m+1}, p=p^{m+1}$ y terminar; en caso contrario:

* Segundo paso (cálculo de la nueva dirección de descenso)

Calcular:

$$
\gamma_{m}=\frac{\left\|g^{m+1}\right\|_{L^{2}(\Omega)}^{2}}{\left\|g^{m}\right\|_{L^{2}(\Omega)}^{2}}
$$

actualizar $w^{m}$ mediante:

$$
w^{m+1}=g^{m+1}+\gamma_{m} w^{m}
$$

luego se toma $\mathbf{u}^{(n+1, m+1)}=\mathbf{u}^{m+1}$ (véase 4.22 ), se hace $m=m+1$ y se regresa a (6.4).

\subsection{Esquema del elemento finito}

El problema (6.3)-(6.11) se resuelve mediante el siguiente esquema discreto del elemento finito, el cual permite determinar, en el caso del método de primer orden y descomposición en dos operadores, para el valor $(n+1) \Delta t$ del tiempo y para cada valor de $m$, los valores de la $(m+1)$-ésima aproximación de la función $\mathbf{u}=\mathbf{u}[x, y,(n+1) \Delta t]$, en los puntos de la malla espacial, a partir de los valores de la $m$-ésima aproximación de esa función: 


$$
\begin{aligned}
& \text { * Paso cero (inicialización) } \\
& \text { Dado } \quad p_{h}^{0} \in H_{h}^{1}, \quad \text { encontrar } \mathbf{u}_{h}^{0} \in \mathbf{V}_{g h} \text {, tal que } \forall \mathbf{v}_{h} \in \mathbf{V}_{0 h}, \\
& a \int_{\Omega} \mathbf{u}_{h}^{0} \cdot \mathbf{v}_{h} d x+\alpha \mu \int_{\Omega} \nabla \mathbf{u}_{h}^{0} \cdot \nabla \mathbf{v}_{h} d x= \int_{\Omega} \mathbf{f}_{h} \cdot \mathbf{v}_{h} d x+\int_{\Omega} p_{h}^{0} \nabla \cdot \mathbf{v}_{h} d x- \\
&-\int_{\Gamma_{1}} \mathbf{g}_{h} \cdot \mathbf{v}_{h} d \Gamma
\end{aligned}
$$

en donde (véase la sección (5)) $\mathcal{T}_{h}$ representa la triangulación de $\Omega$ utilizada para aproximar la prexión, $h$ representa la longitud del mayor lado de los triángulos de $\mathcal{T}_{h}, \mathcal{P}_{k}$ es el espacio de los polinomios de grado menor o igual a $k \mathrm{y}$

$$
H_{h}^{1}=\left\{\psi_{h} \in C^{0}(\bar{\Omega})\left|\psi_{h}\right|_{T} \in \mathcal{P}_{1}, \forall T \in \mathcal{T}_{h}\right\}
$$

es el espacio de funciones utilizado para aproximar la presión. $\widetilde{\mathcal{T}}_{h}$ se define como la triangulación de $\Omega$ que se obtiene, a partir de $\mathcal{T}_{h}$, uniendo los puntos medios de cada uno de sus triángulos y

$$
\mathbf{V}_{h}=\left\{\mathbf{v}_{h}\left|\mathbf{v}_{h} \in C^{0}(\bar{\Omega}) \times C^{0}(\bar{\Omega}), \mathbf{v}_{h}\right|_{T} \in \mathcal{P}_{1} \times \mathcal{P}_{1}, \forall T \in \widetilde{\mathcal{T}}_{h}\right\}
$$

es el espacio de funciones utilizadas para aproximar la velocidad (es decir, la malla de la velocidad es dos veces más fina que la correspondiente a la presión).

Luego, a partir del valor de $\mathbf{u}^{\mathbf{0}}$ así obtenido, definir (solamente en la primera iteración):

$$
g_{h}^{0}=\nabla \cdot \mathbf{u}_{h}^{0} \quad \text { y } \quad w_{h}^{0}=g_{h}^{0}
$$

$\mathbf{y}$, para $m \geq 0$, dados $\left\{p_{h}^{m}, \mathbf{u}_{h}^{m}, g_{h}^{m}, w_{h}^{m}\right\}$ 
calcular $\left\{\overline{\mathbf{u}}_{h}^{m}, \bar{g}_{h}^{m}, p_{h}^{m+1}, \mathbf{u}_{h}^{m+1}, g_{h}^{m+1}, w_{h}^{m+1}\right\}$ como sigue:

* Primer paso (descenso)

Encontrar $\mathbf{u}_{h}^{\mathbf{0}} \in \mathbf{V}_{\mathbf{0 h}}$, tal que $\forall \mathbf{v}_{h} \in \mathbf{V}_{\text {oh }}$

$\alpha \int_{\Omega} \overline{\mathbf{u}}_{h}^{m} \cdot \nabla \mathbf{v}_{h} d x+\mu \int_{\Omega} \nabla \bar{u}_{h}^{m} \cdot \nabla \mathbf{v}_{h} d x=\int_{\Omega} \mathbf{w}_{h}^{m} \nabla \cdot \mathbf{v}_{h} d x$

considerar

$$
\bar{g}_{h}^{m}=\nabla \cdot \overline{\mathbf{u}}_{h}^{m}
$$

y calcular

$$
\begin{gathered}
\rho_{m}=\frac{\int_{\Omega} g_{h}^{m} g_{h}^{m} d x}{\int_{\Omega}\left(a\left|\overline{\mathbf{u}}^{m}\right|^{2}+\mu\left|\nabla \overline{\mathbf{u}}^{m}\right|^{2}\right) d x} \\
p_{h}^{m+1}=p_{h}^{m}-\rho_{m} w_{h}^{m} \\
\mathbf{u}_{h}^{m+1}=\mathbf{u}_{h}^{m}-\rho_{m} \overline{\mathbf{u}}_{h}^{m} \\
g_{h}^{m+1}=g_{h}^{m}-\rho_{m} \bar{g}_{h}^{m}
\end{gathered}
$$

Si $\quad \frac{\left\|g^{m+1}\right\|_{L^{2}(\Omega)}}{\left\|g^{0}\right\|_{L^{2}(\Omega)}}=\frac{\int_{\Omega} g_{h}^{m+1} g_{h}^{m+1} d x}{\int_{\Omega} g_{h}^{0} g_{h}^{0} d x} \leq \epsilon$, entonces tomar $\quad \mathbf{u}_{h}=\mathbf{u}_{h}^{m+1}$, $p_{h}=p_{h}^{m+1}$ y terminar; en caso contrario: 
* Segundo paso (cálculo de la nueva dirección de descenso)

Calcular:

$$
\gamma_{m}=\frac{\int_{\Omega} g_{h}^{m+1} g_{h}^{m+1} d x}{\int_{\Omega} g_{h}^{m} g_{h}^{m} d x}
$$

ctualizar $w_{h}^{m}$ mediante:

$$
w_{h}^{m+1}=\mathbf{g}_{h}^{m+1}+\gamma_{m} w_{h}^{m}
$$

hacer $m=m+1$ y regresar al primer paso.

\section{Resultados}

\subsection{Fluido en una cavidad con pared móvil}

Este problema de prueba se refiere al flujo que ocurre en una cavidad cuadrada con lados de longitud unitaria, cuando su tapa se mueve con velocidad unitaria. Se supone que el fluido está inicialmente en reposo; es decir, se supone $\mathbf{u}(\mathbf{x}, 0)=0$ para todo $x \in \Omega$. En este caso, $\Omega=(0,1) \times(0,1)$, $\Gamma=\{[0,1] \times[0,1]\} / \Omega \quad \mathrm{y}$ las condiciones de frontera son las siguientes:

$$
\mathbf{u}=\mathbf{g}(\mathbf{x}, t)=\left\{\begin{array}{ccc} 
& & \text { si } \quad y=1, \quad 0 \leq x \leq 1 \\
0 & \text { en cualquier otro punto de } \Gamma
\end{array}\right\}
$$

El problema se resolvió para números de Reynolds de 100, 400 y 1000; primero con el método teta y luego con el de primer orden, utilizando una malla de $32 \times 32$ nodos (véase la figura 44) para calcular las presiones y una de $63 \times 63$ nodos para calcular las velocidades. En ambos casos se 
utilizó $\Delta t=0.00005$ y un tiempo total de $t=36$ unidades de tiempo, con lo cual se alcanzó el estado estacionario. En las figuras (2) a la (19) se muestran los diagramas de líneas de flujo, de isobaras y de contornos de velocidad, así obtenidos. La validez de los resultados obtenidos se determinó comparándolos con los reportados en ([1]).

\subsection{Inyección doble en una cavidad cuadrada}

Este segundo problema de prueba se refiere al flujo que ocurre en una cavidad cuadrada con lados de longitud unitaria cuando se inyecta fluido por dos orificios de uno de sus lados y sale fluido por dos orificios del lado opuesto, como se muestra en la figura (45). Los orificios son de 1/16 de ancho, los chorros de entrada tienen una inclinación de $45^{\circ}$, uno está inclinado a la izquierda y el otro a la derecha. Se supone perfiles de velocidad parabólicos y que el fluido está inicialmente en reposo; es decir, se supone $\mathbf{u}(\mathbf{x}, 0)=0$ para todo $x \in \Omega$. Fn este caso, $\Omega=(0,1) \times(0,1)$, $\Gamma=\{[0,1] \times[0,1]\} / \Omega \quad y$ las condiciones de frontera son las siguientes:

$\mathbf{g}\left(x_{1}, x_{2}\right)=\mathbf{0} \quad$ si $\quad x_{1}=0$ ó 1 .

$\mathbf{g}\left(x_{1}, 1\right)=\mathbf{0} \quad$ si $\quad 0 \leq x_{1} \leq 1 / 3, \quad 19 / 48 \leq x_{1} \leq 29 / 48, \quad 2 / 3 \leq x_{1} \leq 1$

$\mathbf{g}\left(x_{1}, 1\right)=-1024\left\{\frac{1}{\sqrt{2}}, \frac{1}{\sqrt{2}}\right\}\left(x_{1}-\frac{1}{3}\right)\left(\frac{19}{48}-x_{1}\right) \quad$ si $\frac{1}{3} \leq x_{1} \leq \frac{19}{48}$

$\mathbf{g}\left(x_{1}, 1\right)=-1024\left\{\frac{1}{\sqrt{2}},-\frac{1}{\sqrt{2}}\right\}\left(\frac{2}{3}-x_{1}\right)\left(x_{1}-\frac{29}{48}\right) \quad$ si $\quad \frac{29}{48} \leq x_{1} \leq \frac{2}{3}$

g $\left(x_{1}, 0\right)=0 \quad$ si $\frac{1}{16} \leq x_{1} \leq \frac{15}{16}$

$\mathbf{g}\left(x_{1}, 1\right)=-1024\left\{0, \frac{1}{\sqrt{2}}\right\} x_{1}\left(\frac{1}{16}-x_{1}\right) \quad$ si $0 \leq x_{1} \leq \frac{1}{16}$

$\mathbf{g}\left(x_{1}, 0\right)=-1024\left\{0, \frac{1}{\sqrt{2}}\right\}\left(1-x_{1}\right)\left(x_{1}-\frac{15}{16}\right) \quad$ si $\frac{15}{16} \leq x_{1} \leq 1$

El problema se resolvió para números de Reynolds de 100,400 y 800 , con el método de descomposición de operadores, utilizando una malla de 
$61 \times 61$ nodos para calcular las presiones y de $121 \times 121$ nodos para calcular las velocidades. Se utilizo $\Delta t=0.0005$ y un tiempo total de $t=12$ unidades de tiempo, con lo cual se alcanzó el estado estacionario. En la figuras (20) a la (26), se muestran los diagramas de líneas flujo, de isobaras y de contornos de vorticidad, así obtenidos. También se analizó, para el caso $R e=800$, la evolución del flujo hasta alcanzar el estado estacionario. Los resultados de ese análisis se muestran en las figuras (27) a (43).

\section{Conclusiones}

Para realizar este trabajo, se implementaron programas de cómputo que permiten resolver las ecuaciones de Navier-Stokes mediante dos métodos de descomposición de operadores: el llamado método teta y un método de primer orden. Para ello, se resolvieron los subproblemas que resultan al aplicar estos métodos, utilizando un método de gradiente conjugado para los problemas de tipo Stokes, y un método de punto fijo para los problemas elípticos de tipo nolineal.

Los programas obtenidos se aplicaron a la simulación del flujo en una cavidad cuadrada con tapa deslizable y al flujo de inyección doble en una cavidad cuadrada. Se encontró que utilizando malla espacial gruesa de $16 \times 16$ nodos, se consigue convergencia con el método teta pero no con el de primer orden. Utilizando una malla gruesa de $32 \times 32$ nodos se consigue convergencia con el método de primer orden.

Se estudió la eficiencia relativa del método de primer orden y del método teta, semicuantitativamente, comparando el tiempo requerido por una PC determinada para resolver hasta alcanzar los estados estacionarios, con cada uno de esos dos métodos, el problema de la cavidad con tapa deslizable. Se encontró que el tiempo requerido por cada uno de ellos es del mismo orden, cuando se corren con la misma malla y con el pismo paso de discretización del tiempo $\Delta t$. Sin embargo, como el método teta puede resolver los problemas con mallas más gruesas que las requeridas por el de primer orden $\mathrm{y}$, por ser de segundo orden, puede resolver los problemas con $\Delta t$ mayor que el requerido por el de prmer orden, el método teta es más eficiente que el de primer orden. La ventaja del método de primer orden radica en que permite descomponer los operadores en más de dos operadores, lo cual no es posible con el método teta. 


\section{Bibliografía}

[1] U. Ghia, K. N. Ghi and C. T. Shin, High-Re Solutions for Incompressible Flow Using the Navier-Stokes and a Multigrid Method, Journal of Computational Physics 48, p. 387-411, 1982.

[2] R. Glowinski, Numerical Methods for Nonlinear Variational Problems, Springer-Verlag, New York, USA, (1984).

[3] R. Glowinski, Finite Element Methods for the Numerical Simulation of Incompressible Viscous Flow Introduction to the Control of the Navier-Stokes Equations, Lectures in App. Math., 28, 219-301, AMS, Providence, RI, (1991).

[4] Claes Johnson, Numerical solution of partial differential equations by the finite element method, Cambridge University Press, Sweden, (1992).

[5] David G. Luenberger, Programación Lineal y No lineal, Addison-Wesley Iberoamericana, México, (1989).

[6] G. I. Marchuk, Methods of Numerical Mathematis, Springer-Verlag, New York, USA, (1975).

[7] K. W. Morton and D. F. Mayers, Numerical solution of partial differential equations, Cambridge University Press, Great Britain, (1994).

[8] David Pnueli and Chain Gutfinger, Fuid Mechanics, Cambridge University Press, U.S.A., (1992).

[9] F. J. Sánchez-Bernabe, On Some Splitting Methods for the Numerical Solutions of the Navier-Stokes Equations, Ph. D. Dissertation, University of Houston, 1996.

[10] F. J. Sánchez-Bernabe, Application of a First Order Operator Splitting Method to Bingham Fluid Flow Simulation, Computers Math. Applic., Vol. 36, No. 3, p. 71-86, 1998. 


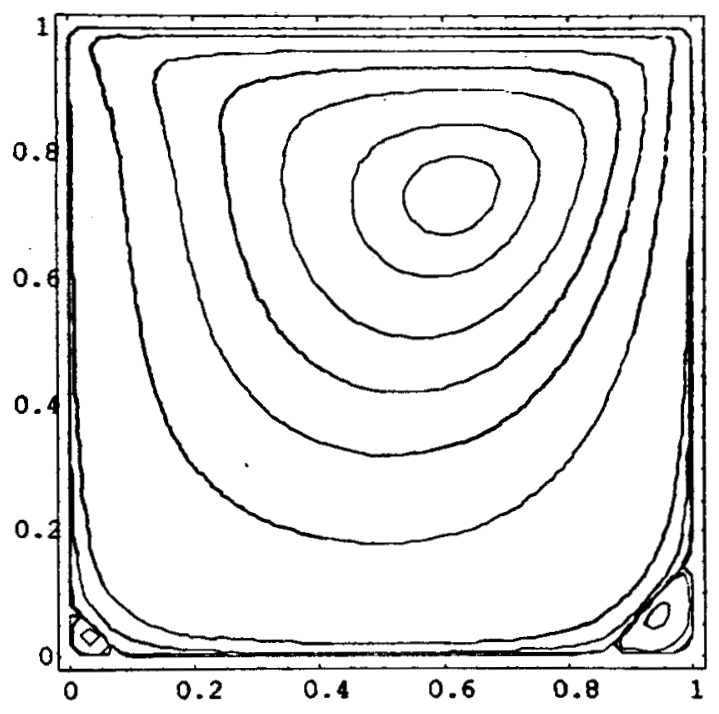

Figure 2: Líneas de flujo obtenidas con el método teta, $R e=100$, malla gruesa de $32 \times 32$ nodos, $\Delta t=0.00005$ y $t=36$.

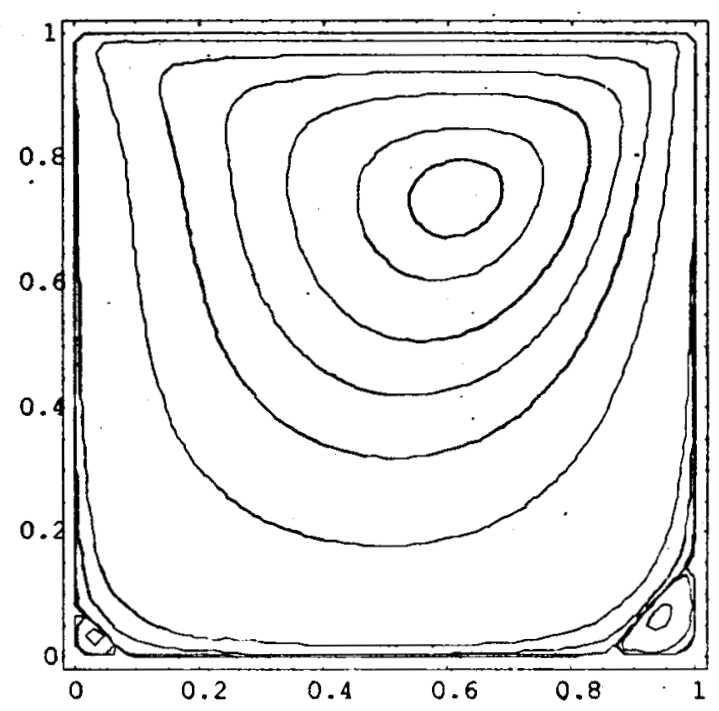

Figure 3: Líneas de flujo obtenidas con el método de primer orden, $R e=100$, malla gruesa de $32 \times 32$ nodos, $\Delta t=0.00005$ y $t=36$. 


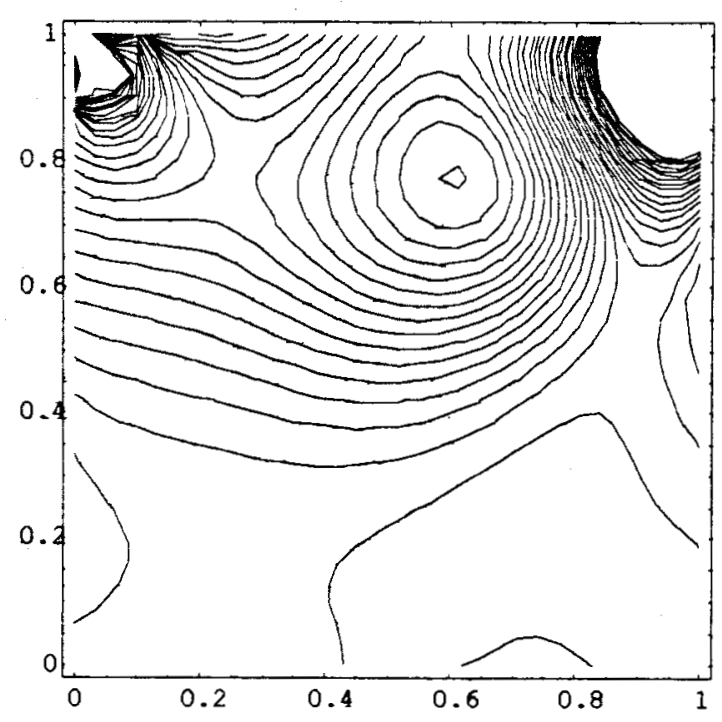

Figure 4:5 Isobaras pbtenidas con el método teta, $R e=100$, malla gruesa de $32 \times 32$ nodos, $\Delta t=0.00005$ y $t=36$.

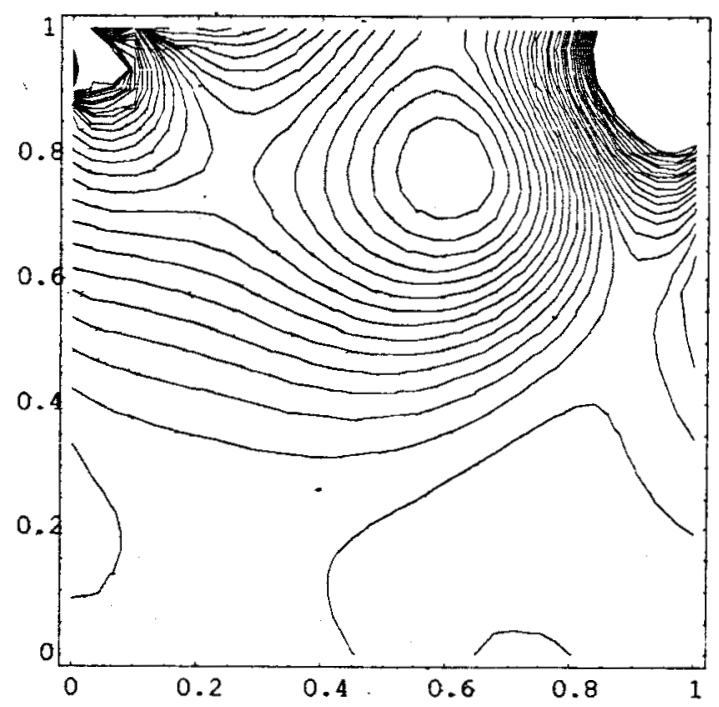

Figure 5: Isobaras obtenidas cop el método de primer orden, $R e=100$, malla gruesa de $32 \times 32$ nodos, $\Delta t=0.00005$ y $t=36$. 


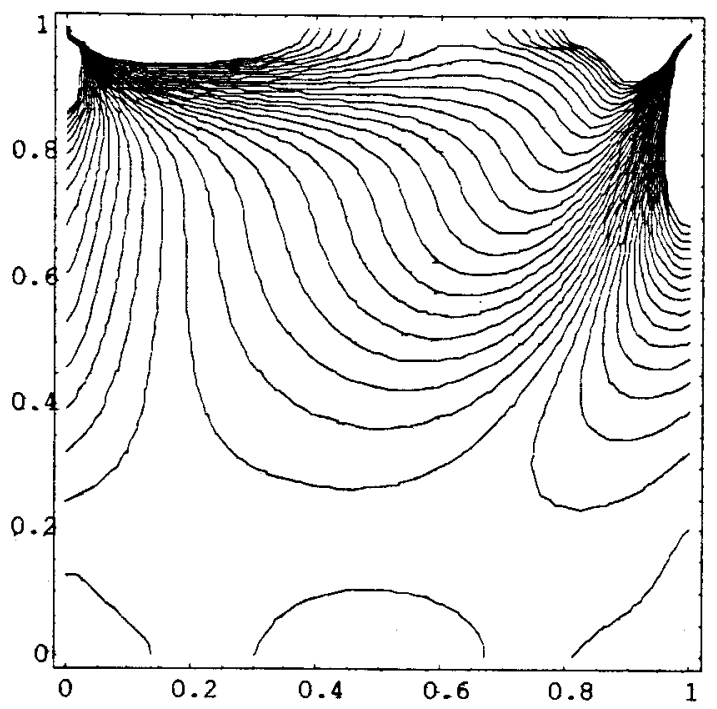

Fìgure 6: Contornos de vorticidad obtenidos con el método teta, $R e=100$, malla gruesa de $32 \times 32$ nodos, $\Delta t=0.00005$ y $t=36$.

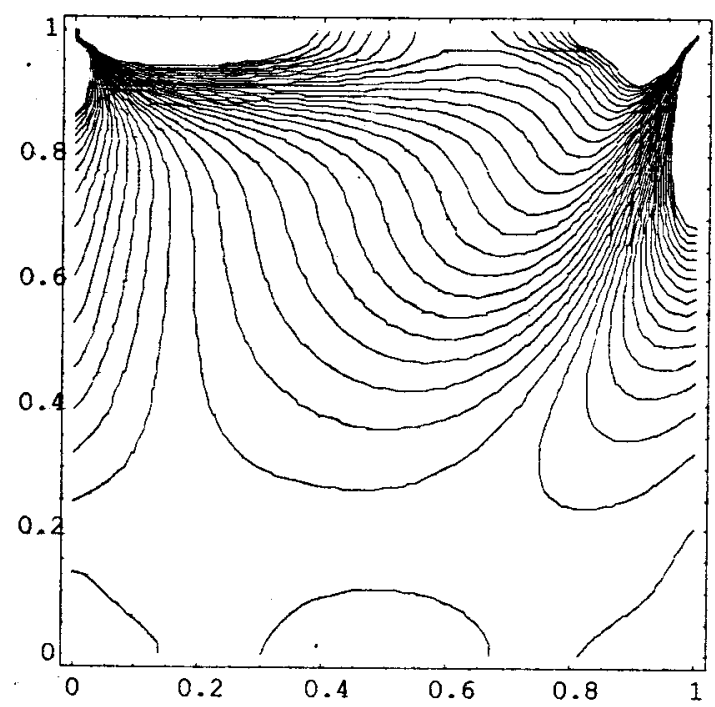

Figure 7: Contornos de vorticidad obtenidos cop el método de primer orden, $R e=100$, malla gruesa de $32 \times 32$ nodos, $\Delta t=0.00005$ y $t=36$. 


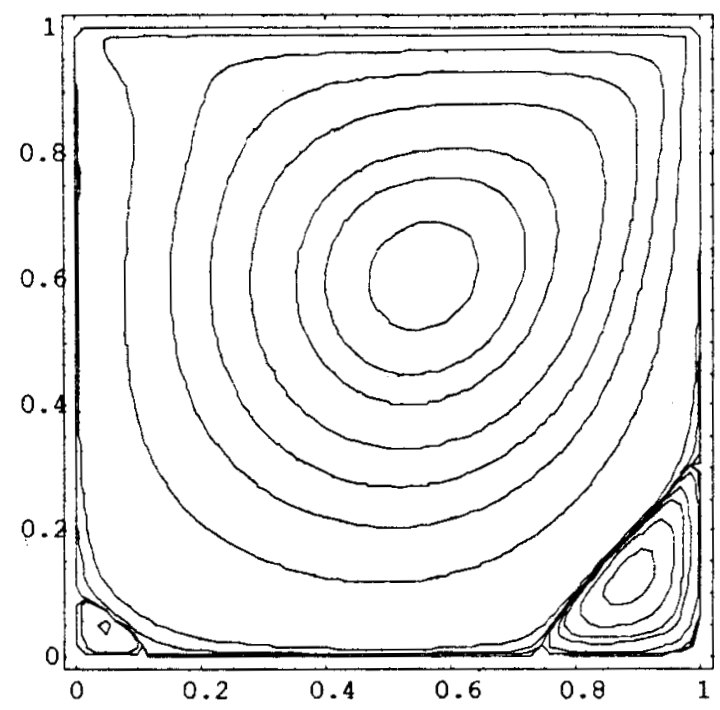

Figure 8: Líneas de flujo pbtenidas con el método teta, $R e=400$, malla gruesa de $32 \times 32$ nodos, $\Delta t=0.00005$ y $t=36$.

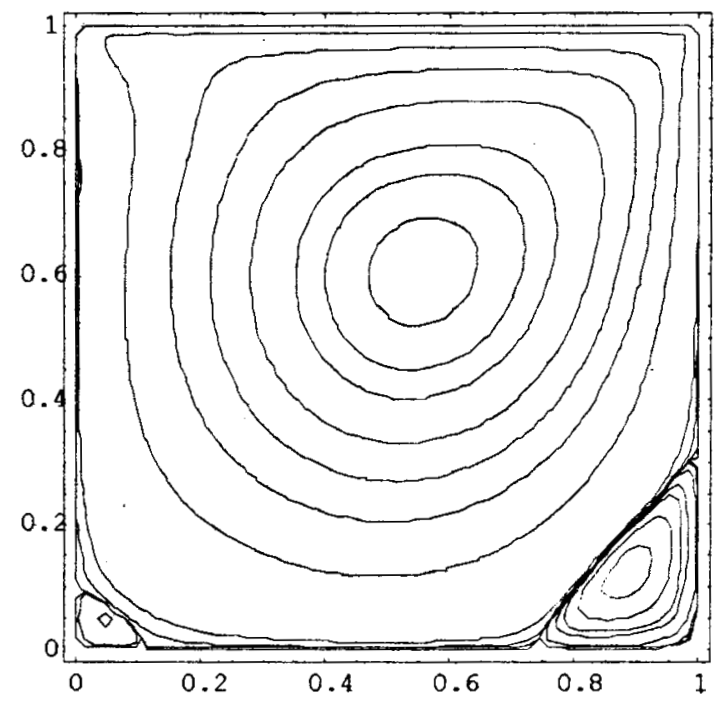

Figure 9: Lîneas de flujo obtenidas con el método de Primer orden, $R e=400$, malla gruesa de $32 \times 32$ nodos, $\Delta t=0.00005$ y $t=36$. 


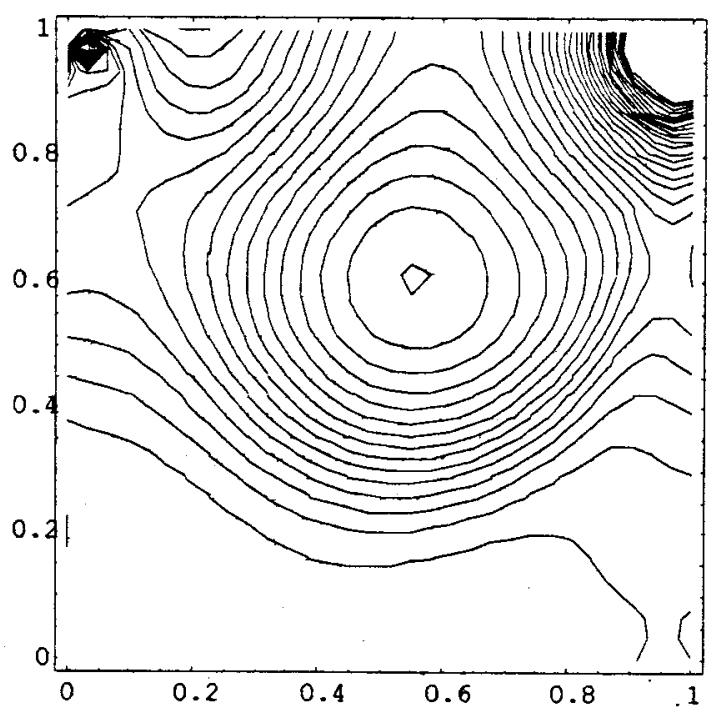

Figure 10: Isobaras pbtenidas con el método teta, $R e=400$, malla gruesa de $32 \times 32$ nodos, $\Delta t=0.00005 \quad$ y $t=36$.

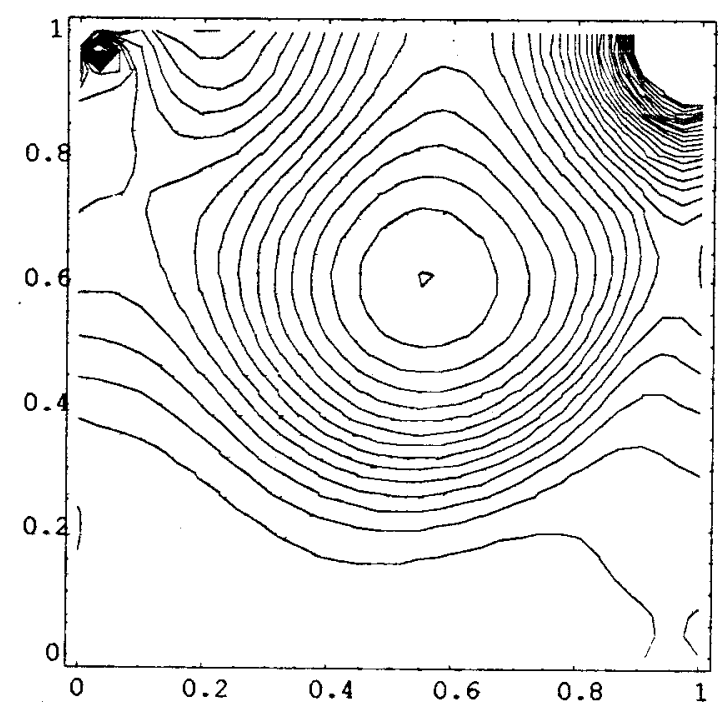

Figure 11: Isonaras obtenidas cop el método de primer orden, $R e=400$, malla gruesa de $32 \times 32$ nodos, $\Delta t=0.00005$ y $t=32$. 


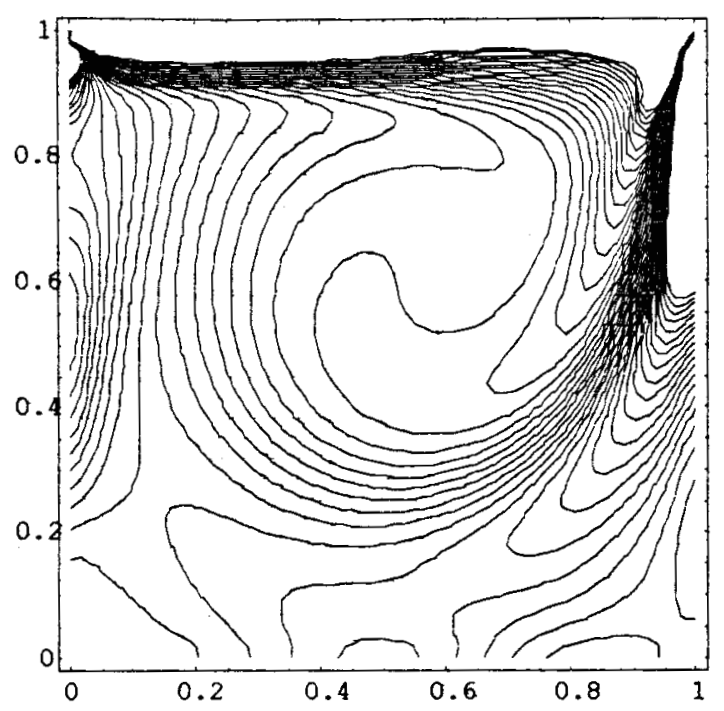

Figure 12: Contornos de vorticidad obtenidos con el método teta, $R e=400$, malla gruesa de $32 \times 32$ nodos, $\Delta t=0.00005$ y $t=36$.

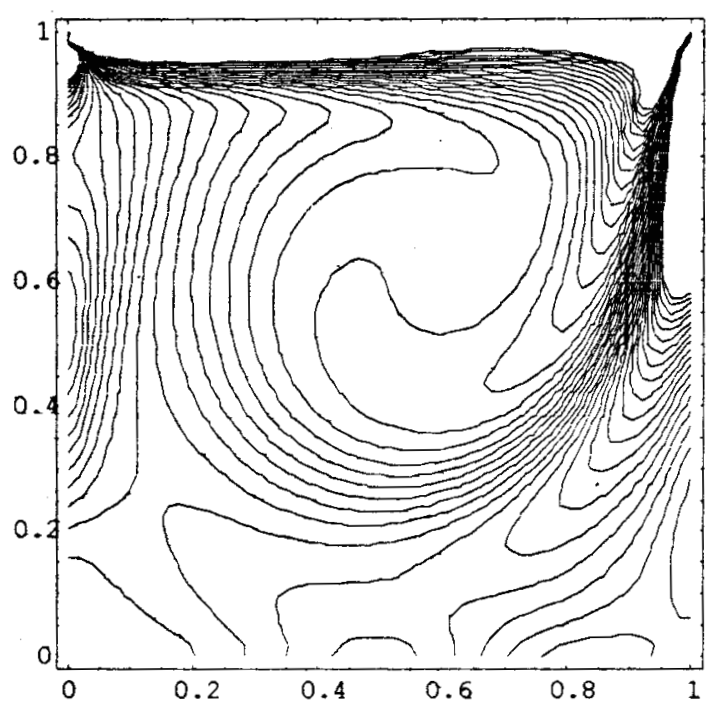

Figure 13: Contornos de vorticidad obtenidos con el método de primer orden, $R e=400$, malla gruesa de $32 \times 32$ nodos, $\Delta t=0.00005 \quad$ y $\quad t=36$. 


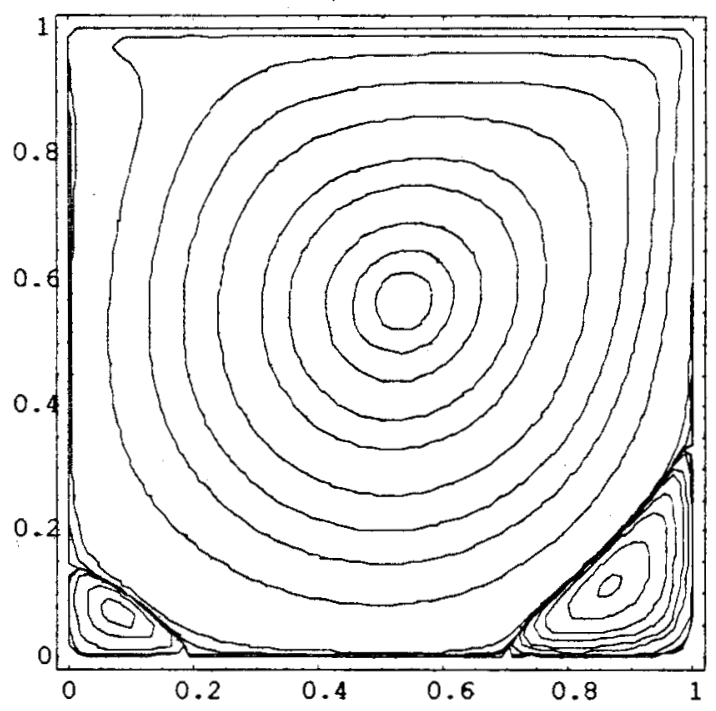

Figure 14: Líneas de flujo qbtenidas con el método teta, $R e=1000$, malla gruesa de $32 \times 32$ nodos, $\Delta t=0.00005$ y $t=36$.

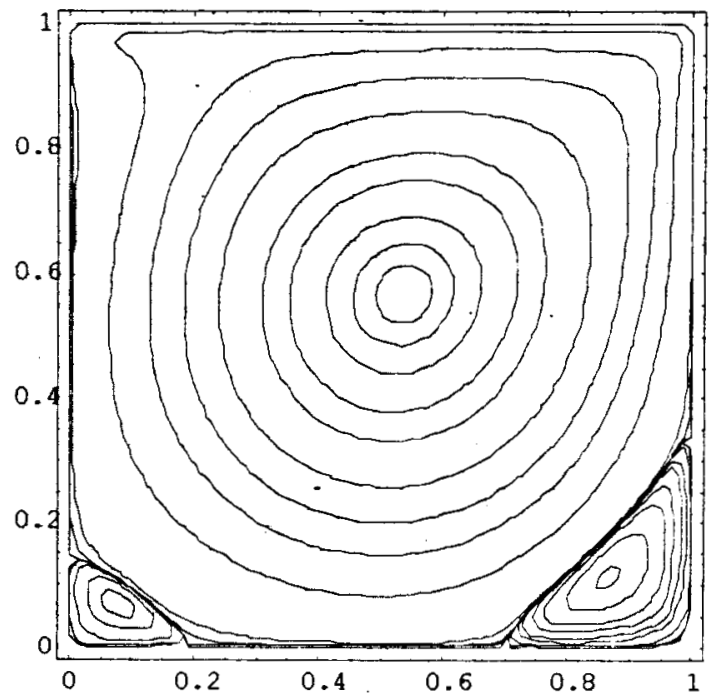

Figure 15: Líneas de flujo obtenidas con el método de primer orden, $R e=1000$, malla gruesa de $32 \times 32$ nodos, $\Delta t=0.00005$ y $t=36$. 


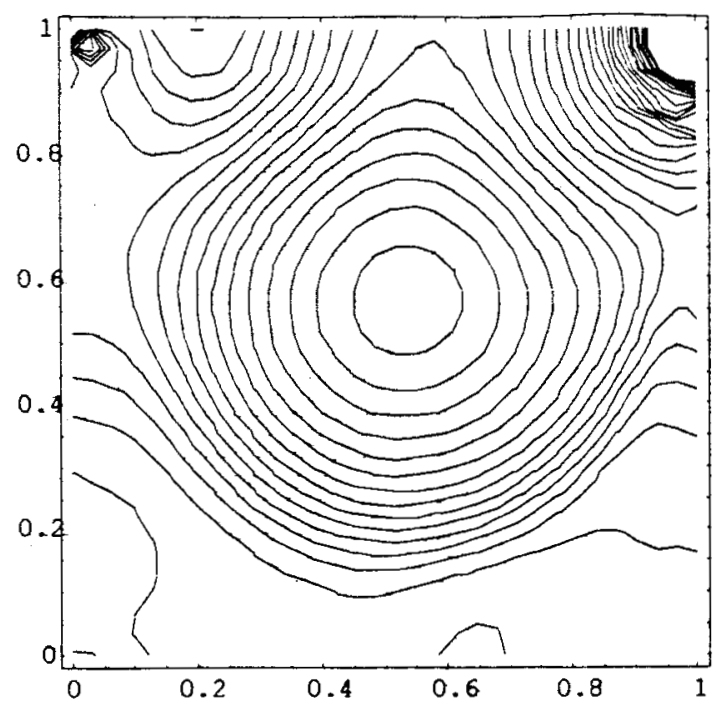

Figure 16: Isobaras obtenidas con el método teta, $R e=1000$, malla gruesa de $32 \times 32$ nodos, $\Delta t=0.00005 \quad$ y $t=36$.

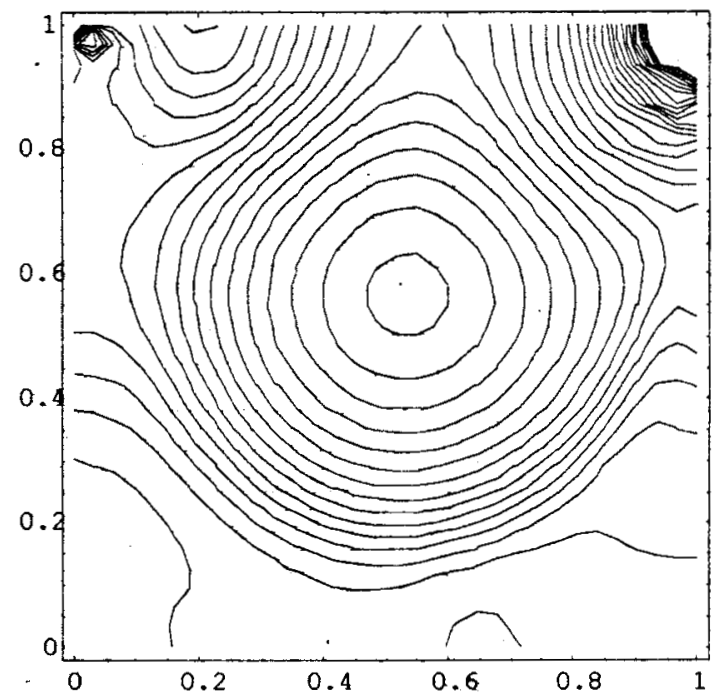

Figure 17: Isobaras obtenidas con el método de primer orden, $R e=1000$, malla gruesa de $32 \times 32$ nodos, $\Delta t=0.00005$ y $t=36$. 


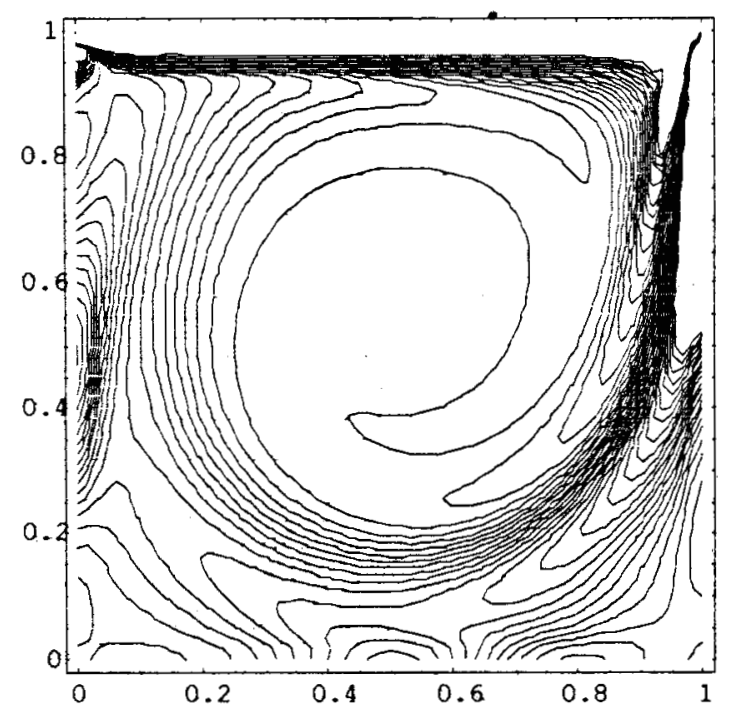

Figure18: Contornos de vorticidad obtenidos con el método teta, $R e=1000$, malla gruesa de $32 \times 32$ nodos, $\Delta t=0.00005$ y $t=36$.

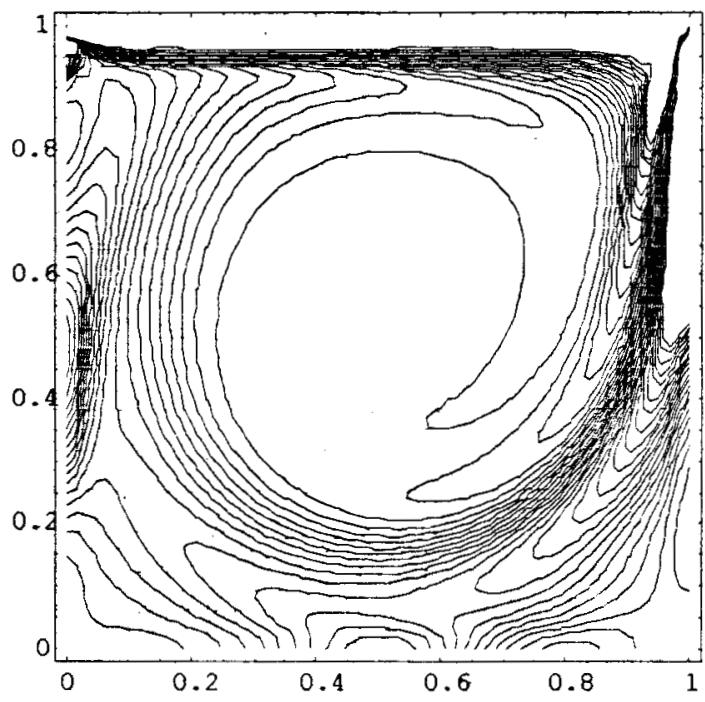

Figure 19: Contornos de vorticidad obtenidos con el método de primer orden, $R e=1000$, malla gruesa de $32 \times 32$ nodos, $\Delta t=0.00005 \quad$ y $\quad t=36$. 


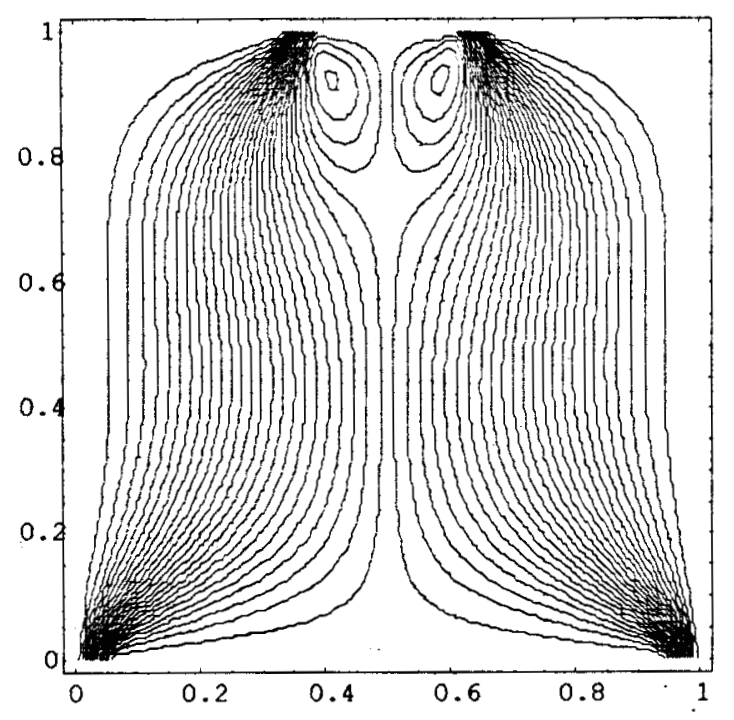

Figure 20: Líneas de flujo obtenidas con el método de primer orden, $R e=100$, malla gruesa de $61 \times 61$ nodos, $\Delta t=0.00005$ y $t=36$.

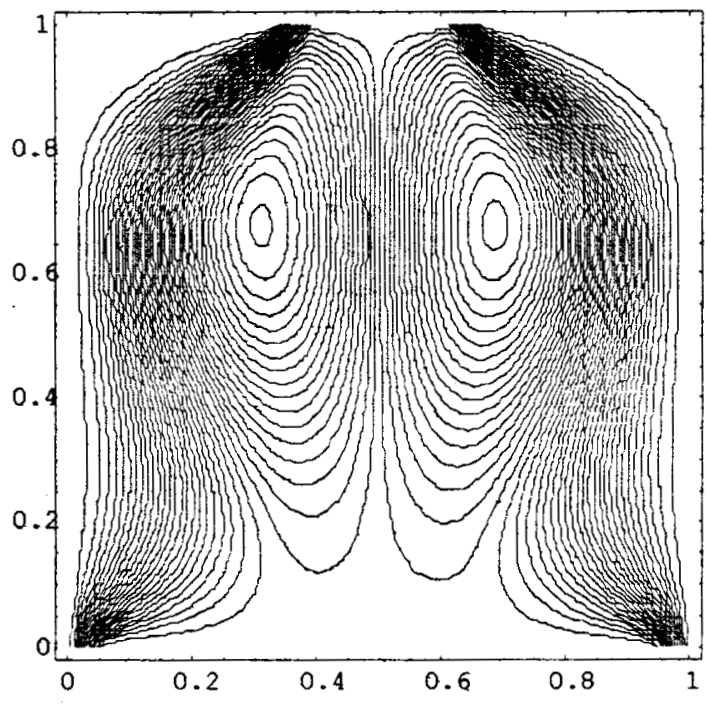

Figure 21: Líneas de flujo obtenidas con el método de primer orden, $R e=400$, malla gruesa de $61 \times 61$ nodos, $\Delta t=0.00005$ y $t=36$. 


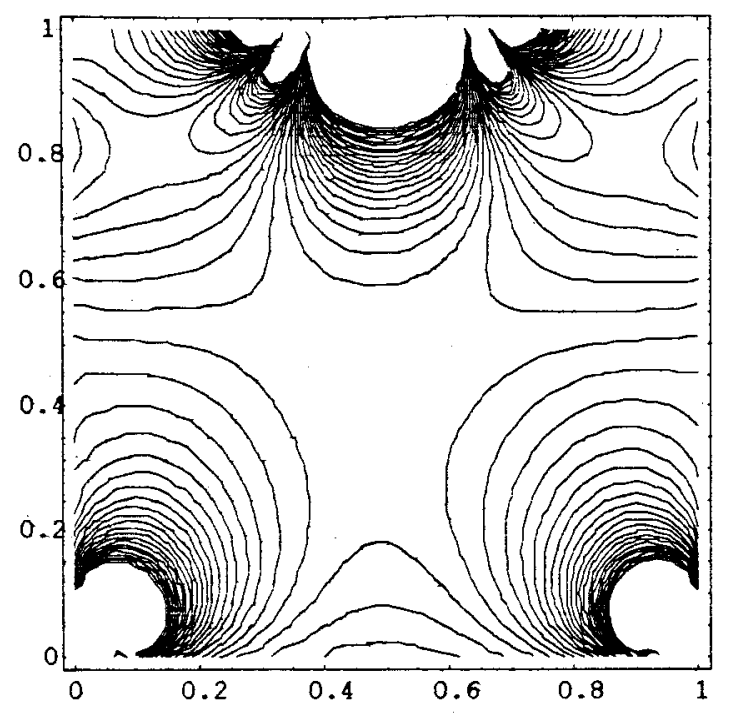

Figure 22: Isobaras obtenidas cop el método de primer orden, $R e=100$, malla gruesa de $61 \times 61$ nodos, $\Delta t=0.00005$ y $t=36$.

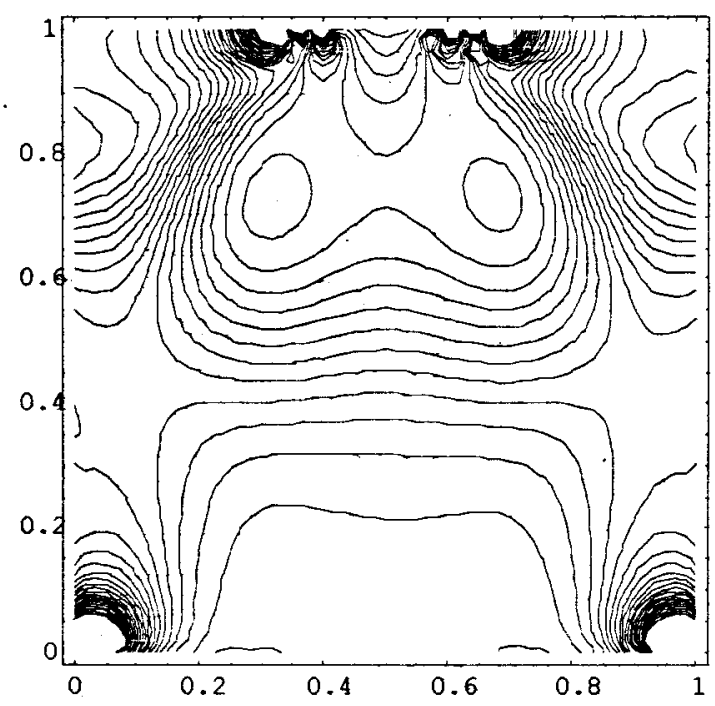

Figure 23: Isobaras obtenidas cop el método de primer orden, $R e=400$, malla gruesa de $61 \times 61$ nodos, $\Delta t=0.00005$ y $t=36$. 


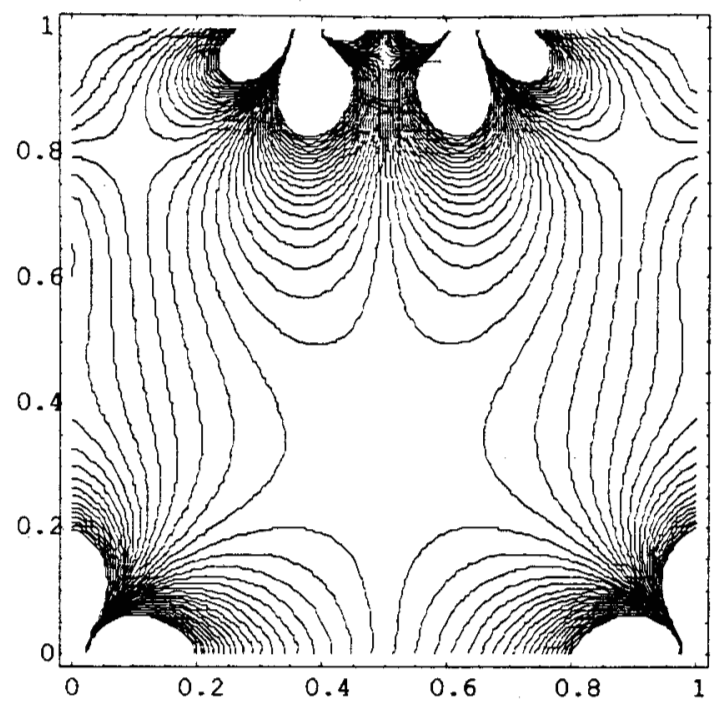

Figure 24: Contornos de vorticidad obtenidos con el método de primer orden, $R e=100$, malla gruesa de $61 \times 61$ nodos, $\Delta t=0.00005$ y $t=36$.

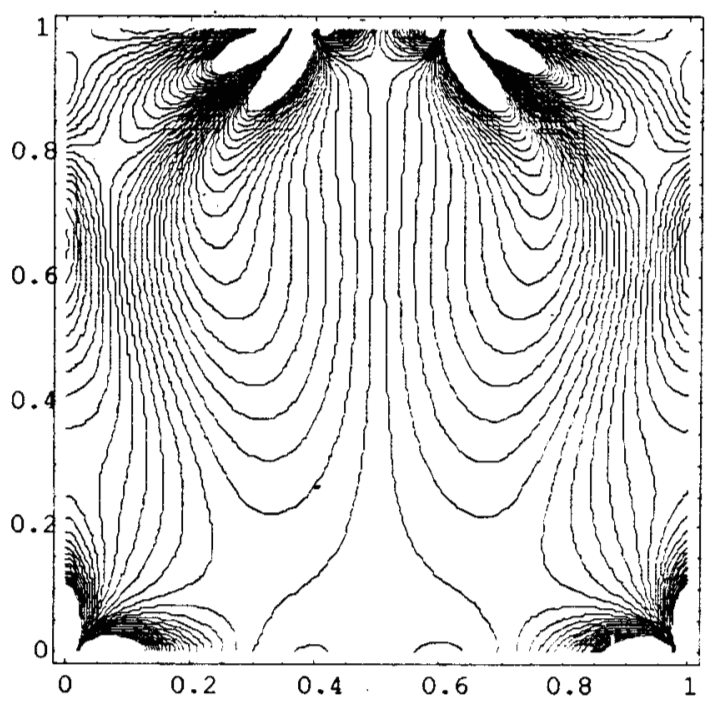

Figure 25: Contornos de vorticidad obtenidos con el método de primer orden, $R e=400$, malla gruesa de $61 \times 61$ nodos, $\Delta t=0.00005$ y $t=36$. 


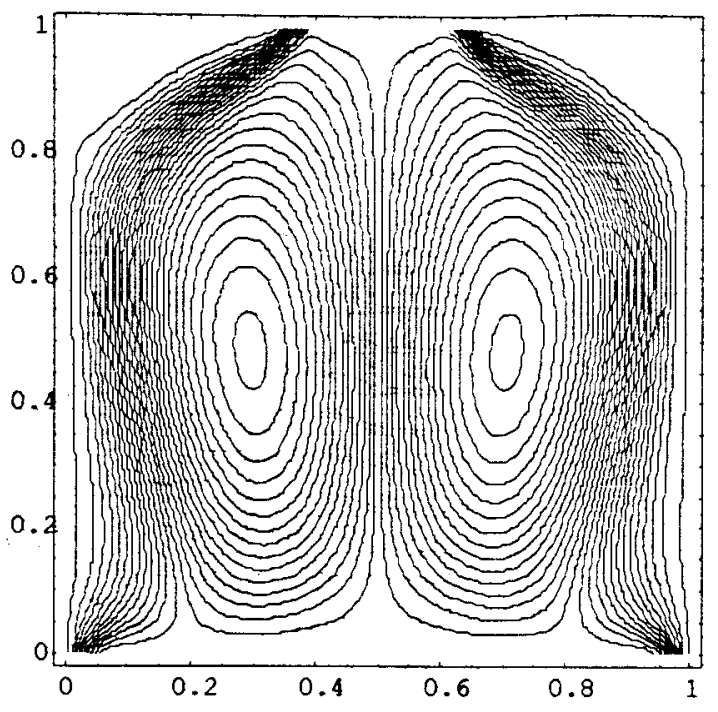

Figure- 26: Líneas de- trajo obtenidas cop el método de primer orden, $R e=800$, malla gruesa de $61 \times 61$ nodos, $\Delta t=0.00005$ y $t=36$.

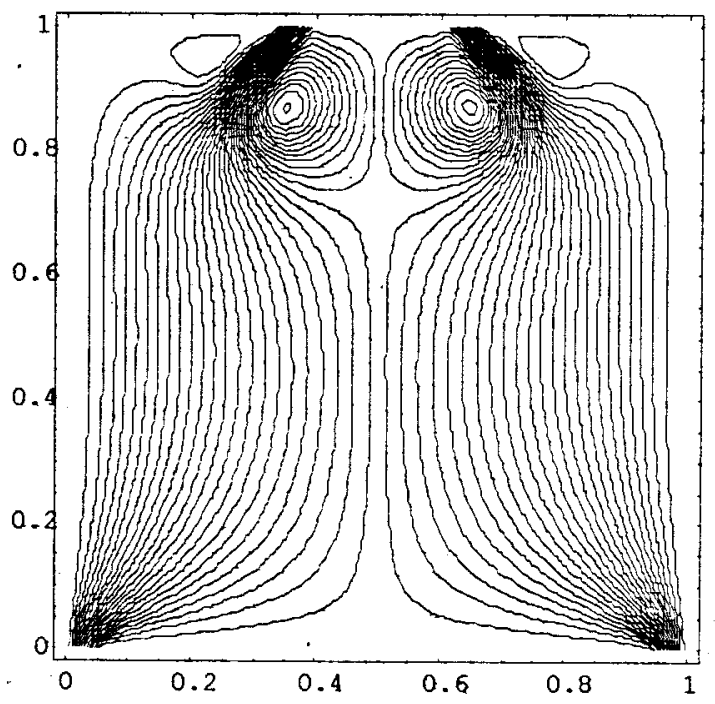

Figure 27: Líneas de flujo obtenidas con el método de primer orden, $R e=800$, malla gruesa de $61 \times 61$ nodos, $\Delta t \mp 0.00005$ y $t=1.5$. 


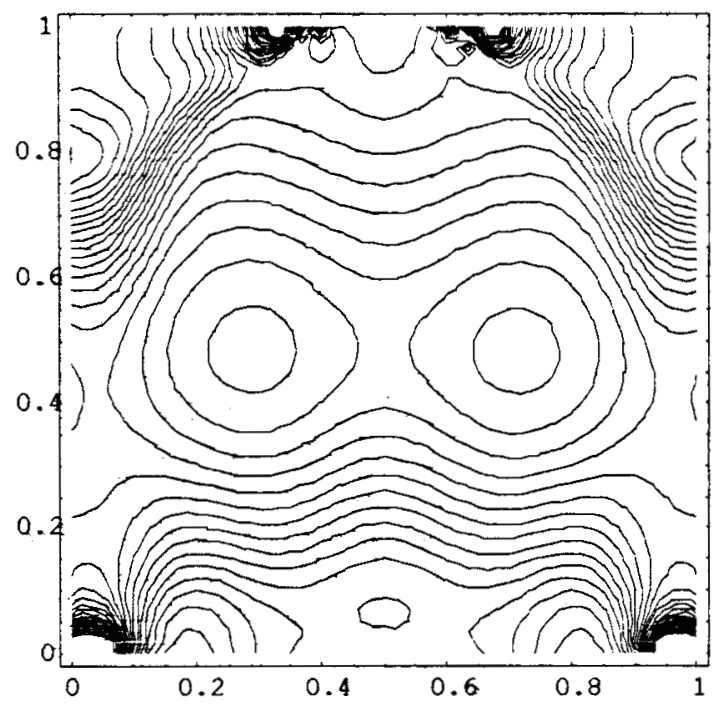

Figure 28: Isobaras obtenidas con el método de primer orden, $R e=800$, malla gruesa de $61 \times 61$ nodos, $\Delta t=0.000 p 5$ y $t=36$.

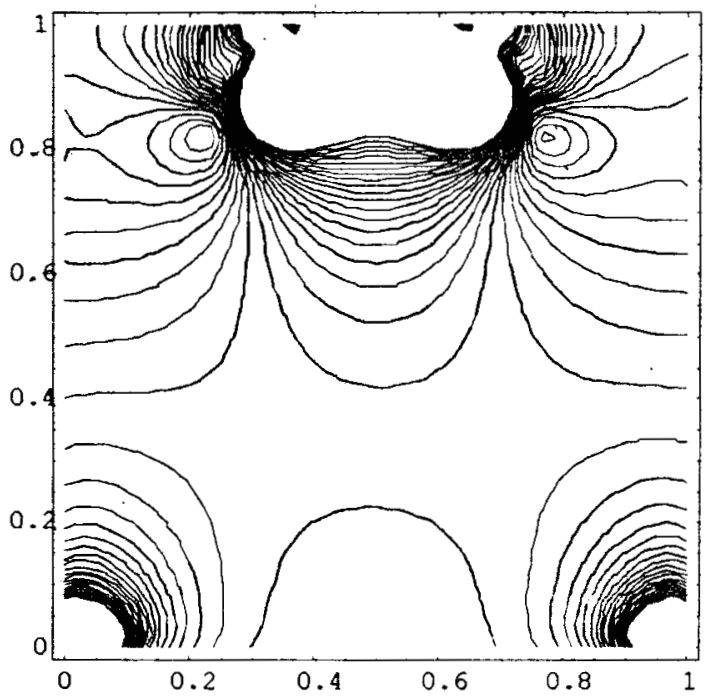

Figure 29: Isobaras obtenidas con el método de prímer orden, $R e=800$, malla gruesa de $61 \times 61$ nodos, $\Delta t=0.00005$ y $t=1.5$. 


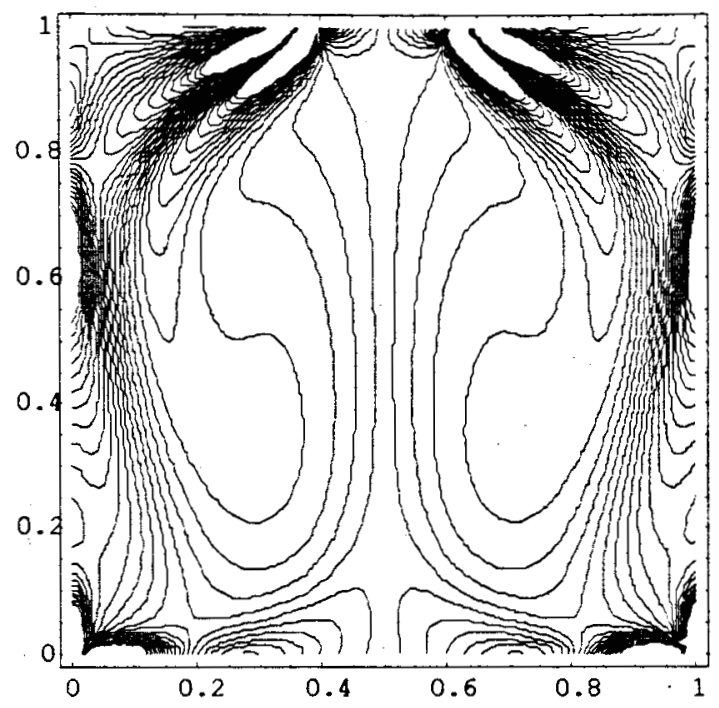

Figure 30: Contornos de vorticidad obtenidos con el método de primer orden, $R e=800$, malla gruesa de $61 \times 61$ nodos, $\Delta t=0.00005$ y $t=36$.

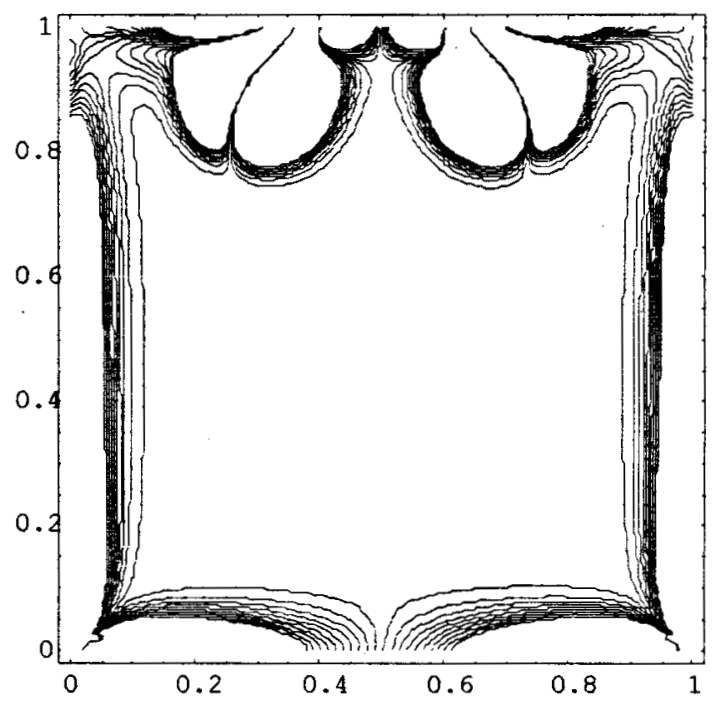

Figure 31: Contornos de vorticidad obtenidos con el método de primer orden, $R e=800$, malla gruesa de $61 \times 61$ nodos, $\Delta t=0.00005$ y $t=1.5$. 


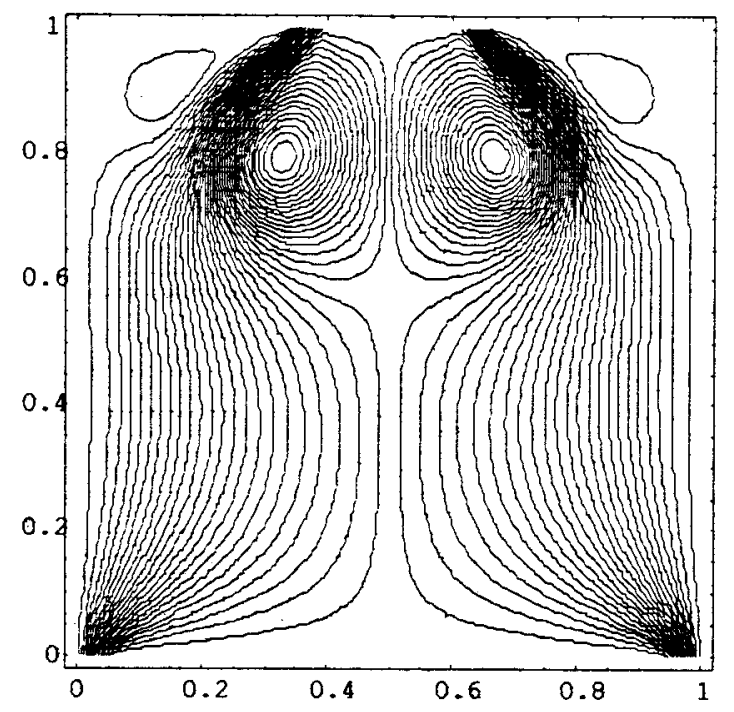

Figure 3z: Eíneas de flajo obtenidas con el método de primer orden, $R e=800$, malla gruesa de $61 \times 61$ nodos, $\Delta t=0.00005$ y $t=3$.

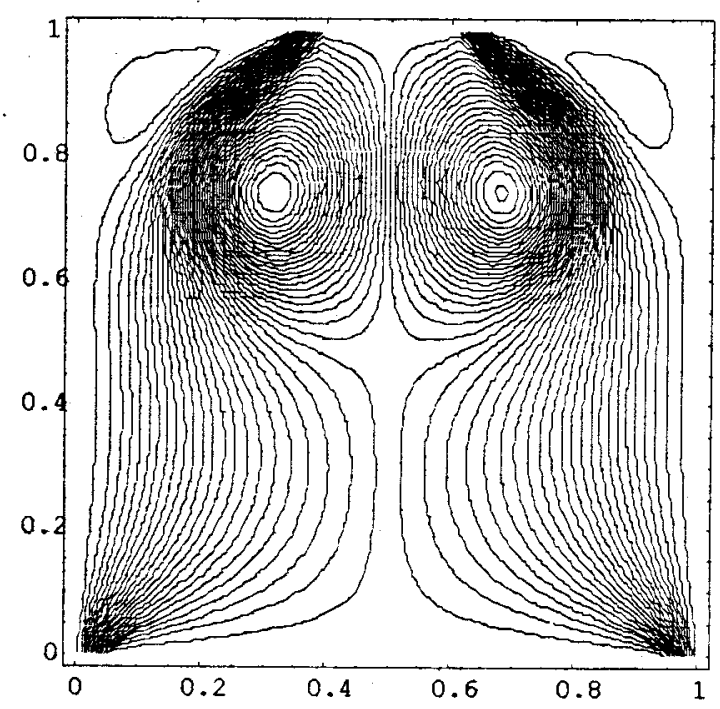

Figure 33: Eíneas de flujo obtenidas con el método de primer orden, $R e=800$, malla gruesa de $61 \times 61$ nodos, $\Delta t \mp 0.00005$ y $t=4.5$. 


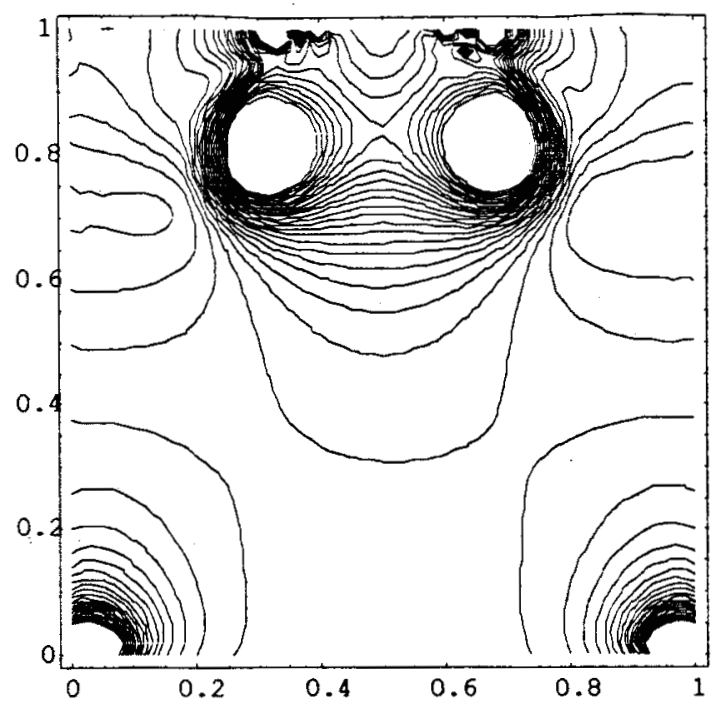

Figure : 34: Isobaras obtenidas con el método de primer orden, $R e=800$, malla gruesa de $61 \times 61$ nodos, $\Delta t=0.00005$ y $t=3$.

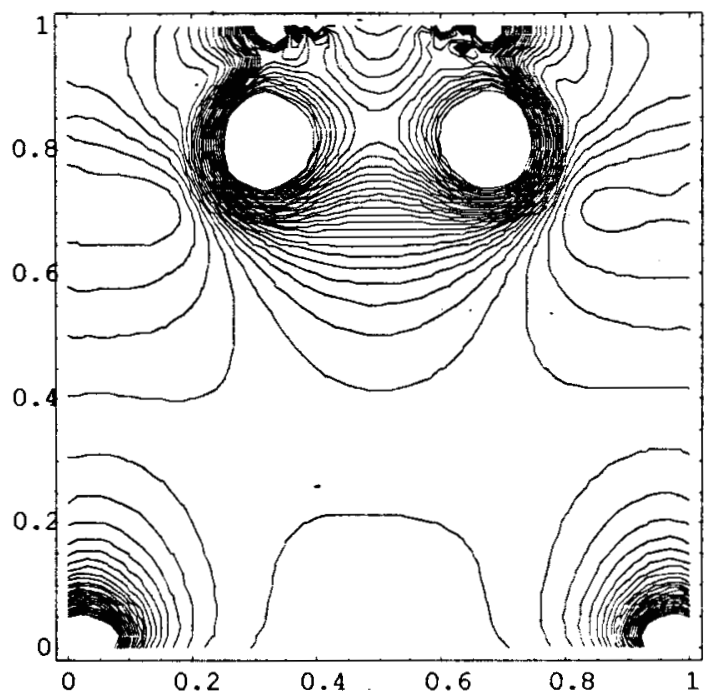

Figure 35: Isobaras obtenidas con el método de primer orden, $R e=800$, malla gruesa de $61 \times 61$ nodos, $\Delta t=0.00005$ y $t=4.5$. 


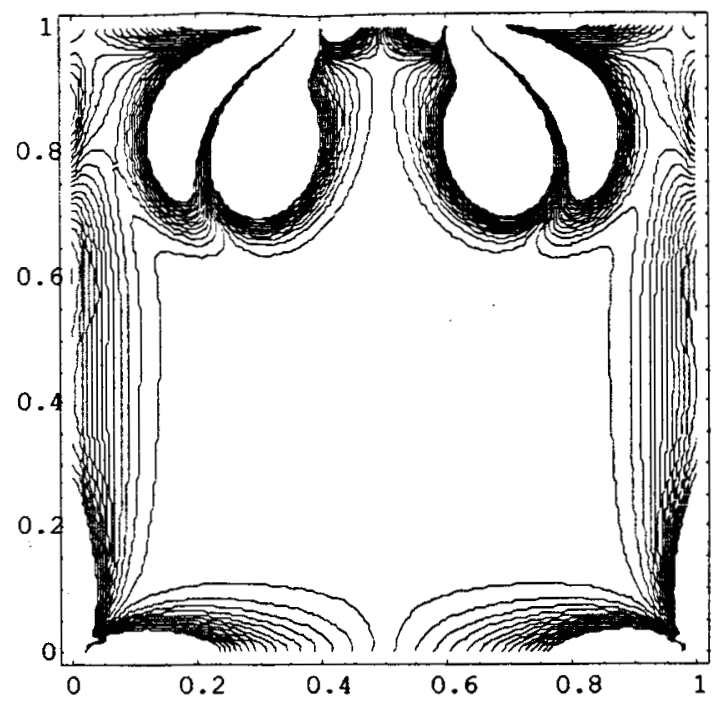

Figure 36: Contornos de vorticidad obtenidos con el método de primer orden, $R e=800$, malla gruesa de $61 \times 61$ nodos, $\Delta t=0.00005$ y $t=3$.

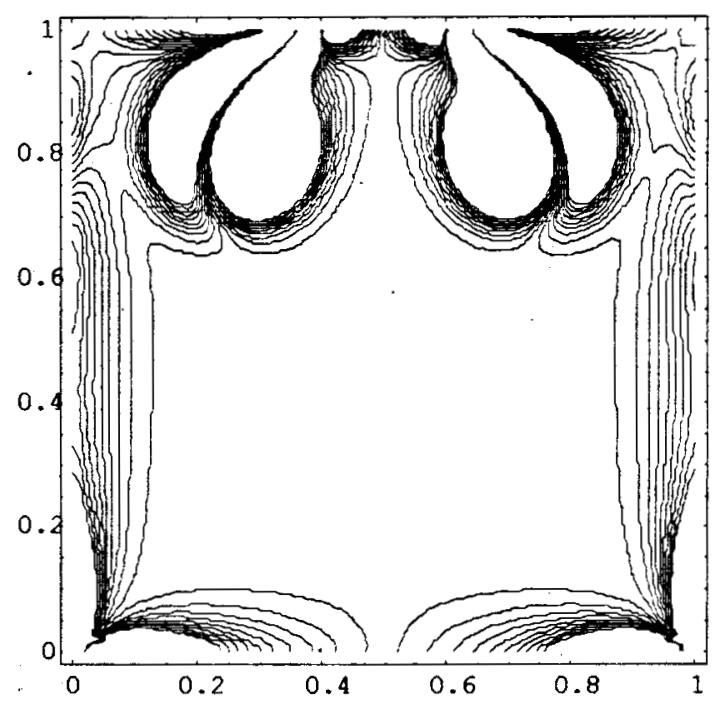

Figure 37: Contornos de vorticidad obtenidos con el método de primer orden, $R e=800$, malla gruesa de $61 \times 61$ nodos, $\Delta t=0.00005$ y $t=4.5$. 


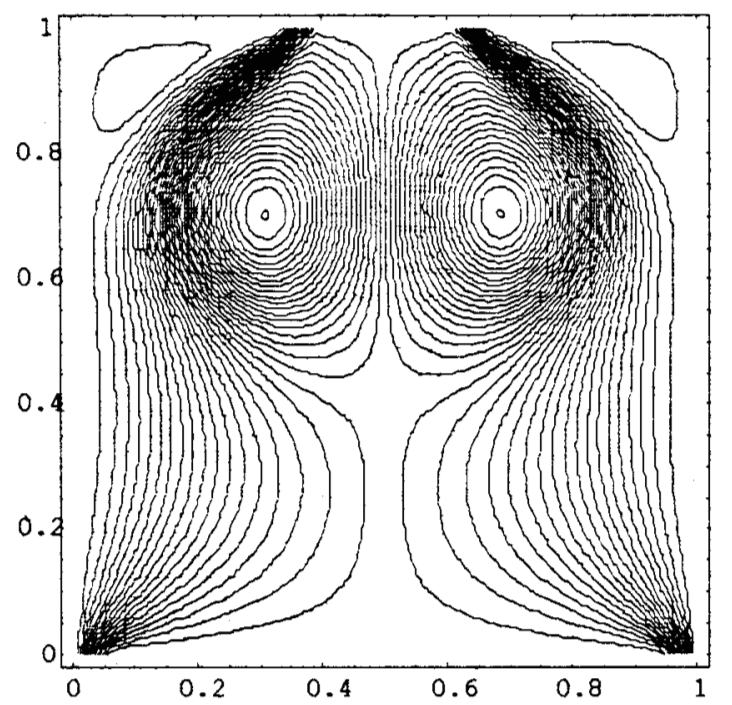

Figure 38: Líneas de flujo obtenidas con el método de primer orden, $R e=800$, malla gruesa de $61 \times 61$ nodos, $\Delta t=0.00005$ y $t=6$.

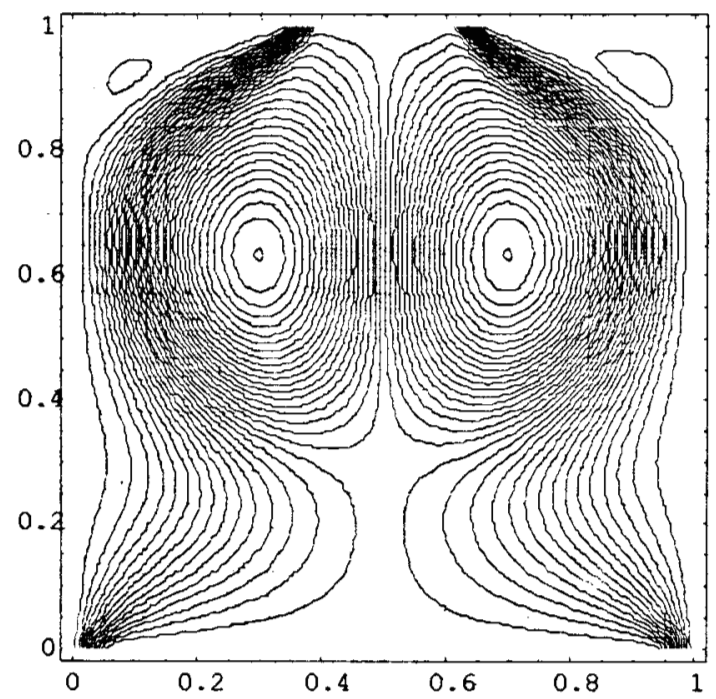

Figture 39: Émeas de flnjo obtenidas con el método de primer orden, $R e=800$, malla gruesa de $61 \times 61$ nodos, $\Delta t=0.00005$ y $t=9$. 


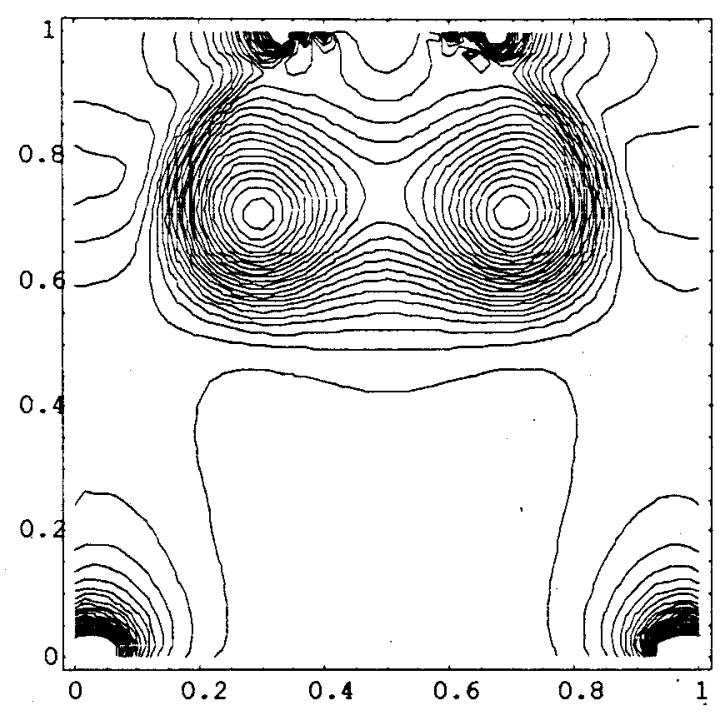

Figure 40: Isobaras obtenidas con el método de primer orden, $R e=800$, malla gruesa de $61 \times 61$ nodos, $\Delta t=0.00005$ y $t=6$.

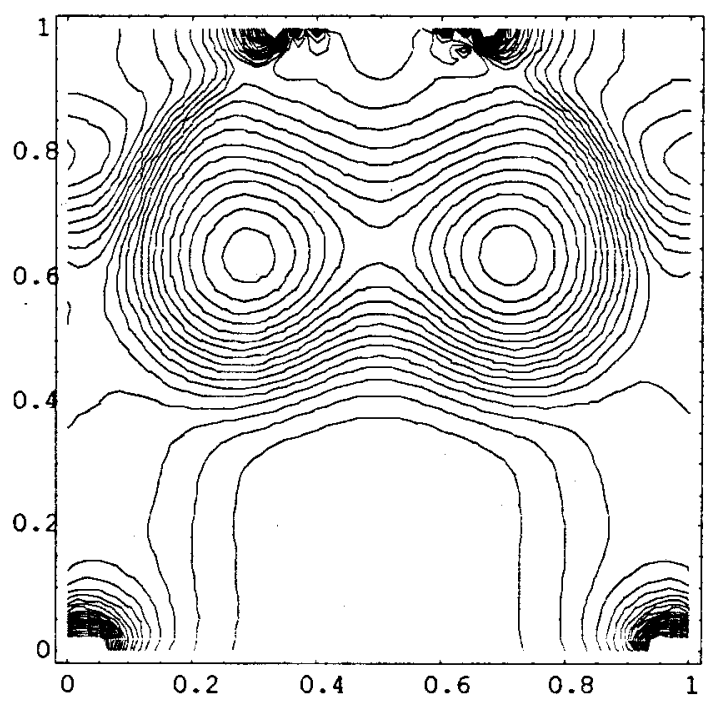

Figure 41: Isobaras obtenidas con el método de primer orden, $R e=800$, malla gruesa de $61 \times 61$ nodos, $\Delta t=0.00005$ y $t=9$. 


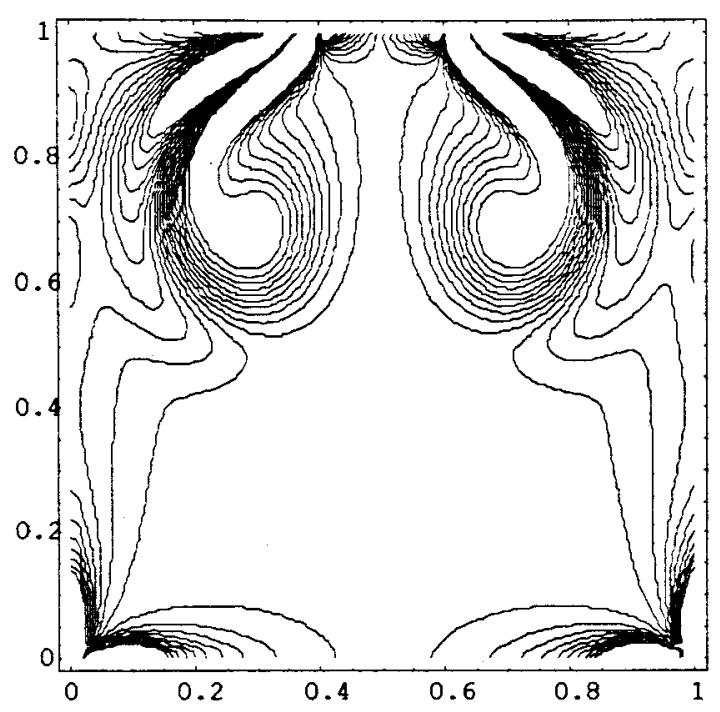

Figure 42: Contornos de vorticidad obtenidos con el método de primer orden, $R e=800$, malla gruesa de $61 \times 61$ nodos, $\Delta t=0.00005$ y $t=6$.

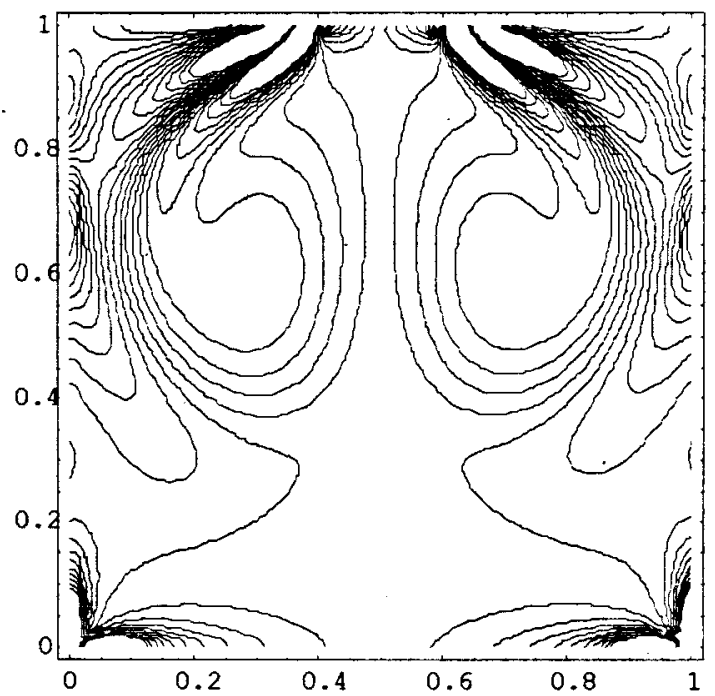

Figure 43: Contornos de vorticidad obtenidos con el método de primer orden, $R e=800$, malla gruesa de $61 \times 61$ nodos, $\Delta t=0.00005 \quad$ y $\quad t=9$. 


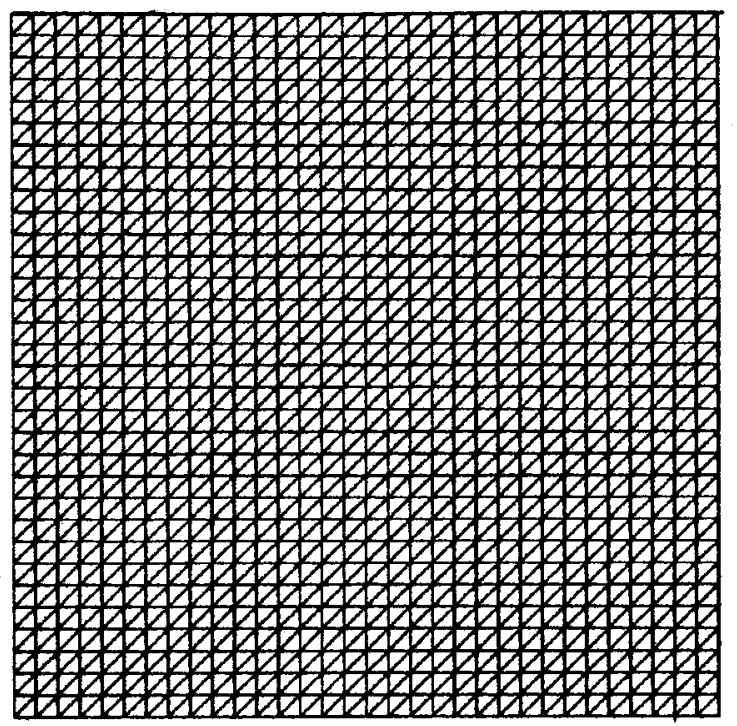

Figure 44 : Malla de $32 \times 32$ Nodos.

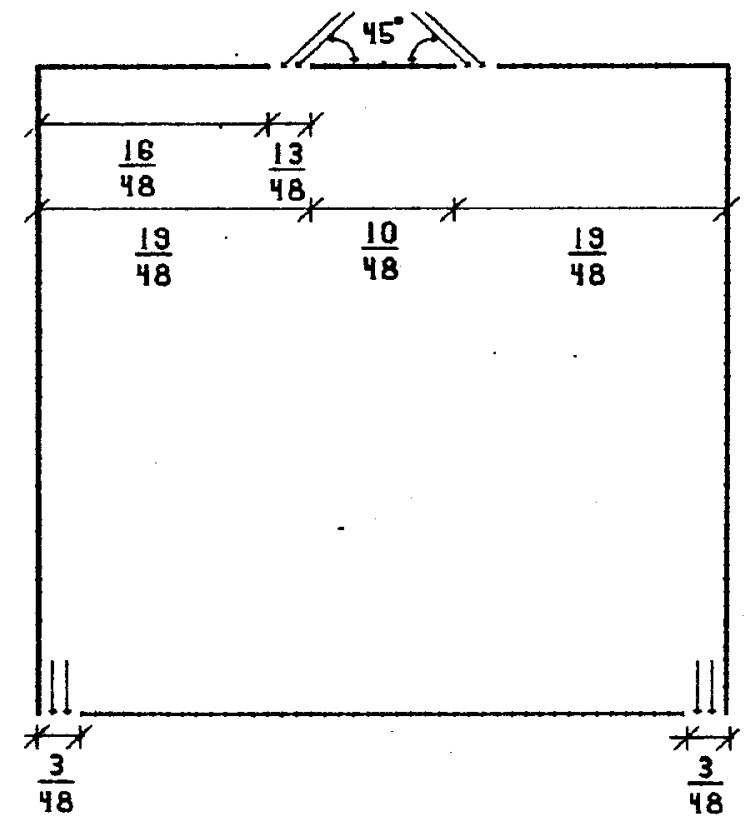

Figure 45: Inyección doble en cavidad cuadrada. 